\title{
Apolipoprotein D mediates autocrine protection of astrocytes and controls their reactivity level, contributing to the functional maintenance of paraquat-challenged dopaminergic systems
}

\author{
Raquel BAJO-GRAÑERAS, Maria D. GANFORNINA*, Esperanza MARTÍN-TEJEDOR \\ and Diego SANCHEZ ${ }^{*}$ \\ Instituto de Biología y Genética Molecular-Departamento de Bioquímica y Biología \\ Molecular y Fisiología, Universidad de Valladolid-CSIC, Valladolid, Spain.
}

* MDG and DS contributed equally to this work.

Running title: ApoD function in the astroglial response to oxidative stress

Abstract word count: 250

Introduction word count: 681

Materials and Methods word count: 1768

Results word count: 3496

Discussion word count: 1385

Bibliography word count: 1600

Legends word count: 1323

Total word count: 10607

Number of figures: 9

Number of tables: 1

Number of Supplementary figures: 6

Number of Supplementary tables: 1

Keywords: lipocalin, oxidative stress, nigrostriatal system

$\S$ Author for correspondence:

Diego Sánchez

Instituto de Biología y Genética Molecular,

c/ Sanz y Forés 3,

Universidad de Valladolid-CSIC,

47003 Valladolid, Spain.

Phone: 983-184814

Fax: 983-184800

e-mail: lazarill@ibgm.uva.es 


\begin{abstract}
The study of glial derived factors induced by injury and degeneration is important to understand the nervous system response to deteriorating conditions. We focus on Apolipoprotein D (ApoD), a Lipocalin expressed by glia and strongly induced upon aging, injury or neurodegeneration. Here we study ApoD function in the brain of wild type and ApoD-KO mice by combining in vivo experiments with astrocyte cultures. Locomotor performance, dopamine concentration, and gene expression levels in the substantia nigra were assayed in mice treated with paraquat (PQ). The regulation of ApoD transcription, a molecular screening of oxidative stress (OS)-related genes, cell viability and oxidation status, and the effects of adding human ApoD were tested in astrocyte cultures.

We demonstrate that: 1) ApoD is required for an adequate locomotor performance, modifies the gene expression profile of PQ-challenged nigrostriatal system, and contributes to its functional maintenance. 2) ApoD expression in astrocytes is controlled by the OS-responsive JNK pathway. 3) ApoD contributes to an autocrine protecting mechanism in astrocytes, avoiding peroxidated lipids accumulation and altering the PQ transcriptional response of genes involved in ROS managing and the inflammatory response to OS. 4) Addition of human ApoD to ApoD-KO astrocytes promotes survival through a mechanism accompanied by protein internalization and modulation of astroglial reactivity. Our data support that ApoD contributes to the endurance of astrocytes and decreases their reactivity level in vitro and in vivo. ApoD function as a maintenance factor for astrocytes would suffice to explain the observed protection by ApoD of OS-vulnerable dopaminergic circuits in vivo.
\end{abstract}




\section{Introduction}

The roles of astrocytes in a healthy central nervous system (CNS) are many and diverse. The already complex known functions of astrocytes, including metabolic support for neurons, local control of blood flow, regulation of extracellular ions and neurotransmitter concentration, have recently been expanded by the discovery of their participation in synaptic information processing and plasticity (Perea et al. 2009). This versatile cell is, in addition, the most resilient cell in the CNS. Astrocytes are particularly durable when our brain ages (Liddell et al. 2010), and are extremely resistant to the many forms of stress that occur when the CNS succumb to disease. Astrocytes respond to CNS disease by a phenotypic transformation known as reactive astrogliosis, which involves a reorganization of their gene expression leading to a profound change in morphology and in migratory and proliferative capacities (Pekny and Nilsson 2005). Astrocyte reactivity can be considered a double-edged sword: triggered as a protection mechanism can become dangerous if out of control. We are starting to comprehend many of the molecular signals and processes that turn-on astrocyte reactivity (Sofroniew 2009). However, the knowledge of mechanisms that restrain the extent of astrogliosis and control its resolution is scarce and fragmentary. The importance of turn-off mechanisms is highlighted by the fact that many pathological situations are caused by defects in processes that must stop glial reactivity.

Oxidative stress (OS), a phenomenon concomitant to most forms of CNS damage and neurodegeneration, is well known to trigger astrocyte reactivity. Oxidative insults are of special concern for dopaminergic systems, since dopamine (DA) metabolism inevitably results in high levels of reactive oxygen species (ROS) that have to be counteracted locally by antioxidant mechanisms (Miller et al. 2009). Astrocytes are therefore clear candidates to play an important 
role not only in the maintenance of dopaminergic systems in the healthy brain, but also in the brain affected by Parkinson's disease (PD), a progressive degenerative disorder primarily characterized by the selective loss of dopaminergic neurons in the substantia nigra (SN). The astroglial response is being studied deeply in PD patients and animal models of PD (Morale et al. 2006; Song et al. 2009), but we are still far from understanding it.

Apolipoprotein D (ApoD), a member of the Lipocalin family secreted by astrocytes and oligodendrocytes, but not expressed in microglia, is known to mediate protective effects for the organism under OS (Ganfornina et al. 2008). The homologues of ApoD in Drosophila, GLaz and NLaz, protect against oxidative damage and contribute significantly to the regulation of longevity (Hull-Thompson et al. 2009; Ruiz et al. 2011; Sanchez et al. 2006; Walker et al. 2006). ApoD is in fact the most robust age dependent up-regulated gene in the brain, conserved across species (de Magalhaes et al. 2009; Loerch et al. 2008), and its expression is boosted by a collection of traumatic, pathological and degenerative nervous system conditions in humans (reviewed by Van Dijk et al. 2006), including Parkinson's disease (Ordonez et al. 2006; Song et al. 2009).

In this work we assay ApoD contribution to the ability of astrocytes to perform protective functions. Our work has three main objectives: (i) to understand how ApoD expression is controlled in the context of the glial response to oxidative insults, (ii) to ascertain whether ApoD contributes to the endurance of astrocytes, therefore potentially contributing to their functional maintenance through aging and disease, and (iii) to test whether ApoD has an impact on the vulnerability of dopaminergic neurons and their functional performance. We use glial cell cultures (primary cortical astrocytes derived from wild type or ApoD-KO mice and human cell lines) as well as mice treated with paraquat (PQ) as our experimental paradigms. 
We have tested whether ApoD null mutant mice have alterations in dopaminergic systems by evaluating locomotor performance, dopamine content, and the molecular response to OS in the substantia nigra. We have assayed whether the stress-activated JNK pathway controls ApoD expression in astrocytes, and how ApoD influences glial viability, their reactivity in vivo and in vitro, and their transcriptional response upon increased OS. Finally, we have tested the ability of exogenous ApoD to improve PQ-challenged astrocyte viability. 


\section{Materials and Methods}

\section{Animals}

Wild type (WT) and loss-of-function mutants for ApoD (ApoD-KO) mice were bred at the University of Valladolid animal facility. Genotyping was performed by PCR as previously described (Ganfornina et al. 2008). Mice were fed standard rodent chow and water ad libitum in ventilation-controlled cages in a $12 \mathrm{~h}$-light/dark cycle. Experimental cohorts used in this study are the F1 generation of homozygous crosses of ApoD-/- and ApoD+/+ littermates born from heterozygous crosses of the ApoD-KO line in C57BL/6J background. This strategy avoids potential maternal effects of ApoD and generates WT and ApoD-KO cohorts with homogeneous genetic background.

Experimental procedures were approved by the University of Valladolid Animal Care and Use Committee in accordance with the Guidelines for the Care and Use of Mammals in Research (European Commission Directive 86/609/CEE, Spanish Royal Decree 1201/2005).

\section{PQ and LPS treatments}

Male mice ( 8 months old, $\mathrm{N}=6$ /genotype for $\mathrm{PQ}$ and $\mathrm{N}=4$ /genotype for carrier) were injected intraperitoneally with either $10 \mathrm{mg} \mathrm{kg}^{-1} \mathrm{PQ}$ or PBS for a total of 7 injections (twice a week for the first 2 weeks, 1/week for 3 additional weeks) and used for locomotor activity tests, dopamine measures, immunoblot and qRT-PCR analyses. Open field behavioral tests were performed 6 days after the last injection and tissues obtained 7 days after the last injection. A second cohort (7 months old males, $\mathrm{N}=4$ /genotype for $\mathrm{PQ}$ and $\mathrm{N}=2$ /genotype for carrier) was used for immunohistochemistry after the same protocol of PQ injections. 
Two 7 months old WT male mice injected with a single dose of $30 \mathrm{mg} \mathrm{kg}^{-1}$ PQ or $3.3 \mathrm{mg} \mathrm{kg}^{-1}$ LPS for 12 hours were used as controls for acute effects of oxidative and pro-inflammatory stimuli.

\section{Locomotor activity}

Open field tests were carried out with a MIR-100 infrared digital camera and the Activity Monitor (v. 5.0) acquisition and analysis program (Med Associates). Mice locomotor behavior was explored during a 5 min session.

\section{HPLC determination of dopamine and its catabolites}

Dopamine (DA) and its catabolites (4-dihydroxy-phenylacetic acid, DOPAC, and homovanillic acid, HVA) were measured in the brain portion anterior to the substantia nigra. Brain tissue was homogenized in $0.1 \mathrm{~N}$ perchloric acid, $0.1 \mathrm{mM}$ EDTA, and centrifuged ( $8 \mathrm{~min}, 1000 \mathrm{~g})$. Supernatants (50 $\mu 1$ aliquots) were injected into an HPLC system equipped with a Phenomenex Gemini 5 C18 (particle size $5 \mu \mathrm{m}$ ) column (mobile phase: $\mathrm{Na}_{2} \mathrm{HPO}_{4} 25 \mathrm{mM}$, sodium octane sulphonate $0.6 \mathrm{mM}$, EDTA $0.1 \mathrm{mM}, 10 \%$ methanol, $\mathrm{pH} 4.35)$. DA, DOPAC and HVA concentrations, expressed as pmol/mg of tissue, were estimated using reference standards (Sigma).

\section{Immunocytochemistry}

Cells attached to poly-L-lysine treated coverslips were fixed with $4 \%$ formaldehyde in PBS. Following washes in PBS, blocking and permeabilization (TritonX-100 0.25\% in PBS, $1 \%$ normal goat serum), cells were incubated with either rabbit serum anti-GFAP (Dako, Denmark) or rabbit serum anti-hApoD (generated by C. López-Otín). Cy3 or Cy2-conjugated goat anti-rabbit IgG 
(Abcam, UK) were used as secondary antibodies for fluorescence immunocytochemistry. After washes in PBS, preparations were mounted with Vectashield-DAPI (Vector Labs). Labeled cells were visualized with an Eclipse 90i (Nikon) fluorescence microscope equipped with a DS-Ri1 (Nikon) digital camera. Images were acquired under the same conditions of illumination, diaphragm and condenser adjustments, exposure time, background correction and color levels, and processed with NIS-Elements BR 3.0 software (Nikon) for fluorescence quantification. A minimum of five 20x fields were quantified.

Confocal images were obtained in a DMI 6000B microscope with a TCS SP5 X confocal system and a WLL laser (Leica) controlled by LAS AF software (Leica).

\section{Immunohistochemistry}

Mouse brains were quickly removed and midbrains cut horizontally in $200 \mu \mathrm{m}$ sections on a vibratome (Microm). Slices were fixed in 4\% paraformaldehyde for $3 \mathrm{~h}$ at room temperature, washed, and blocked for $2 \mathrm{~h}$ (in $0.5 \%$ triton X-100, $1 \%$ normal goat serum) before overnight incubation at $4{ }^{\circ} \mathrm{C}$ with either mouse serum anti- tyrosine hydroxylase (TH) (Sigma) or rabbit serum anti-GFAP (Dako, Denmark) antibodies. Following extensive washes with PBS slices were incubated with secondary antibody (Goat anti-mouse Cy3 or anti-rabbit Alexa 488 (Santa Cruz, $\mathrm{CA}$ ) for $2 \mathrm{~h}$ at room temperature. Labeled cells were visualized in an Eclipse 90i (Nikon) as described above.

TH-positive cells from the SN pars compacta $(\mathrm{SNc})$ were counted in the areas described by McCormack et al. (2002) in 7 slices per mouse (Fig. S1). GFAP fluorescence quantification was performed in the same slices (see Fig. S1 for the areas selected) as described above in the immunocytochemistry section. 


\section{Immunoblot analysis}

Brain tissue, either from SN or from striatum, was homogenized in lysis buffer (1\% Nonidet P-40, 0.1\% SDS, 10\% Glycerol, 1\% sodium deoxycholate, $1 \mathrm{mM}$ Dithiothreitol, $1 \mathrm{mM}$ EDTA, $100 \mathrm{mM}$ HEPES, $100 \mathrm{mM} \mathrm{KCl,} \mathrm{10 \%} \mathrm{Complete} \mathrm{Protease} \mathrm{Inhibitors} \mathrm{(Roche)} \mathrm{in} \mathrm{PBS),} \mathrm{centrifuged} \mathrm{after} 30$ min at $4{ }^{\circ} \mathrm{C}$, and the supernatant stored at $-80^{\circ} \mathrm{C}$. Cultured cells were lysed in the same buffer. Protein concentration was determined with Micro-BCA ${ }^{\mathrm{TM}}$ protein assay (Pierce). Immunoblot analyses were performed with 10-20 $\mu \mathrm{g}$ of total protein/lane transferred to PVDF membranes using standard procedures. We used the following primary antibodies: Rabbit serum anti-GFAP (Dako, Denmark); Rabbit serum anti-hApoD (generated by C. López-Otín); Rabbit anti-Sod2 (Santa Cruz, CA); Goat anti-HO-1 (Santa Cruz, CA); and Goat serum anti-mApoD (Santa Cruz, CA). Secondary HRP-conjugated Goat anti-Rabbit or Donkey anti-goat IgG (Santa Cruz, CA) were used. Each blot was also incubated with HRP-conjugated anti- $\beta$ actin antibody (Sigma) for normalization purposes. Membranes were developed with ECL (Millipore) and the integrated optical density of the immunoreactive protein bands was measured in images taken within the linear range of the camera (VersaDoc, BioRad) avoiding signal saturation. Mean \pm SD of arbitrary density units was calculated from at least duplicate blots.

\section{Cell cultures}

Primary cortical glial cultures. The cerebral cortices of neonatal (P0) mice were quickly extracted. The meninges were removed by rolling on a sterile filter paper, and pieces of cortex were placed in Earle's Balanced Salt Solution (EBSS) containing $2.4 \mathrm{mg} / \mathrm{ml}$ DNAse I and $0.2 \mathrm{mg} / \mathrm{ml}$ bovine serum albumin. The tissue was minced with a surgical blade, centrifuged ( $200 \mathrm{~g}, 2 \mathrm{~min})$, incubated 
with $10 \mathrm{mg} / \mathrm{ml}$ trypsin for $15 \mathrm{~min}$ at $37^{\circ} \mathrm{C}$ (incubation terminated by addition of $10 \% \mathrm{FBS}$ ), mechanically dissociated with a Pasteur pipette, and centrifuged (200g, $5 \mathrm{~min})$. The last two steps were repeated, and the resulting cells were resuspended in Dulbecco's Modified Eagle's medium (DMEM) with 10\% FBS, 1\% L-Gln, 1\% P/S/A (Penicillin (10U/ $\mu \mathrm{l})$ - Streptomycin $(10 \mu \mathrm{g} / \mu \mathrm{l})$ Amphotericyn B $(25 \mu \mathrm{g} / \mathrm{ml}))$. Cells were plated onto culture flasks and incubated at $37^{\circ} \mathrm{C}$ in $5 \%$ $\mathrm{CO}_{2}$ with $90-95 \%$ humidity. Medium was weekly replaced. After $2-3$ subculture steps, over $95 \%$ of type 1 astrocytes were present, as estimated by GFAP labeling and by morphological criteria. Cultures had a minor contribution of microglial cells, but oligodendrocytes were not detected.

The cell lines 1321N1, HeLa, and HEK were grown and maintained in DMEM with 5\% FBS, $1 \% \mathrm{~L}-\mathrm{Gln}$ and $1 \% \mathrm{P} / \mathrm{S} / \mathrm{A}$ at $37^{\circ} \mathrm{C}$ in $5 \% \mathrm{CO}_{2}$ with $90-95 \%$ humidity.

\section{Quantitative RT-PCR}

RNA from homogenized mesencephalic fragments or cultured cells were extracted with TRIzol (Invitrogen). Total RNA $(1 \mu \mathrm{g})$ was reverse-transcribed with PrimeScript ${ }^{\mathrm{TM}}$ (Takara) and treated with DNaseI. The cDNA obtained was used as template for qRT-PCR amplifications. We used TaqMan probes for the genes Gdnf, Alox15, Nos2, Il6, Tnfa, Mbp and ApoE (Primers and probes designed by Roche Applied Science; Universal ProbeLibray). To amplify mouse and human ApoD we used SYBR Green I (Takara) and the following primers. Mouse Rpl18-Forward: 5'TTCCGTCTTTCCGGACCT; Mouse Rpl18-Reverse: 5'- TCGGCTCATGAACAACCTCT; Mouse ApoD-Forward: 5'- GAAGCCAAACAGAGCAACG; Mouse ApoD-Reverse: 5'TGTTTCTGGAGGGAGATAAGGA; Human PL18-Forward: CCATCATGGGAGTGGACAT; Human PL18-Reverse: 5'- CACGGCCGTCTTGTTTTC; Human ApoD-Forward: 5'CCACCCCAGTTAACCTCACA; Human ApoD-Reverse: 5'- CCACTGTTTCTGGAGGGAGA. 
Rpl18 was used as the reference gene because neither genotype nor treatment gives a significant fold change for this gene.

To study genes related to the antioxidant response and ROS metabolism, we used SYBR Green and the qRT-PCR array PMM-065A (SABiosciences). Gapdh was selected as reference gene. Amplifications were performed in quadruplicate in an ABI Prism 7900HT or a Rotor-Gene RG3000 (Corbett Research) thermal cycler. Standard cycling conditions were: $95^{\circ} \mathrm{C}, 5 \mathrm{~min} ; 40 \mathrm{x}$ $\left(95^{\circ} \mathrm{C}, 30 \mathrm{sec} ; 60^{\circ} \mathrm{C}, 1 \mathrm{~min}\right)$.

Changes in transcriptional expression were estimated with the $\Delta \Delta C_{T}$ method (Livak and Schmittgen 2001). The following criteria were applied to our analysis: 1) Replicates with variation coefficient $>2.5 \%$ were excluded. 2) Undetermined $\mathrm{C}_{\mathrm{T}}$ values (gene expression below detection levels) were assigned $\mathrm{C}_{\mathrm{T}}=35$. 3. Pairwise comparisons where the gene average $C_{T}>35$ cycles in both conditions were excluded from the analysis. 4) Only transcriptional changes $\geq$ twofold were included in the analysis. Significant differences of gene transcriptional changes were evaluated with a Mann-Whitney U-test, using $\Delta \mathrm{C}_{\mathrm{T}}$ of each replica. Values are expressed as mean $\pm \mathrm{SEM}$. Only statistically significant $(p<0.05)$ differences of expression are presented and discussed in the text.

\section{Viability assay}

Astrocyte viability was measured by the extent of 3-(4,5-Dimethylthiazol-2-yl)-2,5-

diphenyltetrazolium bromide (MTT; Sigma) reduction to insoluble intracellular formazan (dependent on the activity of intracellular dehydrogenases). Cultures were incubated with MTT (5 $\mathrm{mg} / \mathrm{ml}$ in PBS) for $3 \mathrm{~h}$ at $37^{\circ} \mathrm{C}$. An equal volume of solubilization buffer (2-Propanol $0.04 ; 0.1 \mathrm{~N}$ $\mathrm{HCl} ; 10 \%$ TritonX-100) was added and mixed thoroughly to dissolve the formazan crystals. MTT 
reduction was measured spectrophotometrically by subtracting background at $690 \mathrm{~nm}$ from the absorbance at $570 \mathrm{~nm}$ and expressed as \% control.

\section{Apoptosis assays}

Flow-cytometry analysis. Cells were treated with trypsin-EDTA, washed with PBS, resuspended in $100 \mu \mathrm{l}$ Annexin V binding buffer, and incubated with $4 \mu \mathrm{l}$ of FITC-conjugated Annexin V and $4 \mu 1$ propidium iodide (PI) according to the manufacturer's specifications (Immunostep). Cells were incubated for $30 \mathrm{~min}$ at room temperature in the dark and diluted with $400 \mu$ of Annexin V binding buffer just before injection into a flow cytometer (Gallios; Beckman Coulter). Data were analyzed with the Kaluza software (v1.1; Beckman Coulter).

TUNEL labeling kit (Roche) was used to assay apoptotic cell death both in mesencephalic tissue sections and in primary glial cell cultures. Immunolabeled cells were observed with a Nikon (Eclipse 80i) microscope and a DS-Ril digital camera. Images were acquired and processed with the NIS-Elements BR 3.0 software (Nikon).

\section{Biochemical assays}

TBARS assay. Brain tissue was homogenized in PBS in the presence of butylated hydroxytoluene (BHT). Extracts were incubated with $0.2 \mathrm{M}$ glycine-HCl, pH 3.6 and TBA reagent $(0.5 \%$ TBA, $0.5 \%$ SDS). After 15 min incubation at $90^{\circ} \mathrm{C}$, samples were cooled on ice and transferred to a 96 well microplate for triplicate readings. Absorbance was monitored at $532 \mathrm{~nm}$ in a Versamax microplate reader (Molecular Devices).

Malondialdehyde concentration (MDA-586 assay, Bioxytech), and aconitase activity (Aconitase-340 assay, Bioxytech) were measured following the manufacturer's recommendations. 
In all these assays the experimental values were normalized to protein concentration, measured with the Micro BCA Protein Assay (Pierce). At least two independent experiments with measurements in triplicate were performed.

\section{Statistical analysis}

Statistical analyses were performed with Statgraphics plus (v 5.0) software. $p<0.05$ was defined as a threshold for significant changes. 


\section{Results}

\section{ApoD-KO mice show alterations in the dopaminergic system associated with bradykinesia upon chronic paraquat treatment.}

We have previously demonstrated that ApoD confers protection at the organism level, promoting survival and preventing brain tissue oxidation upon different paradigms of PQ intraperitoneal injections (Ganfornina et al. 2008). Moreover, ApoD contributes to the early response to OS, as it is transiently up-regulated in the brain of mice acutely exposed to PQ (Ganfornina et al. 2008). The present study was designed to examine whether ApoD has protective potential for the nigrostriatal dopaminergic system, a neuronal circuit particularly vulnerable to OS and markedly sensitive to PQ toxicity. PQ and other neurotoxicants have been used as chemical models of Parkinson's disease. We used a paradigm of PQ injections (see Methods) that generates a mild OS and slow PQ accumulation in the brain (Prasad et al. 2009), but not systemically (Prasad et al. 2007). With this low-dose chronic PQ exposure, ApoD mRNA levels in the WT substantia nigra (SN), measured 7 days after the last injection, do not differ from control sham-injected mice (Fig. S2A), revealing that the induction of ApoD mRNA expression has already resolved and returned to basal levels by the end of the treatment. However, as expected from the stability of the Lipocalin fold, ApoD protein is maintained at slightly higher levels than the control samples after this chronic PQ treatment (Fig. S2B). Therefore, the genotype-dependent changes we describe below will be the consequence of the absence of transient peaks of ApoD expression after each PQ injection (as revealed by the acute treatments used as positive controls in Fig. S2B), and of the constant mild increase of this stable extracellular protein. 
As expected for this low-dose chronic PQ treatment, no significant differences in open field activity are observed in WT mice 6 days after the $7^{\text {th }}$ PQ dose (Fig. 1A). However, ApoD-KO mice show a significant PQ-dependent decrease in locomotor activity (bradykinesia), demonstrating that without ApoD the functional circuits controlling motor outputs become more vulnerable to the long-term effects of PQ.

To analyze the functional state of dopaminergic systems, brains were studied 7 days after the $7^{\text {th }}$ PQ dose. It is known that alterations in DA levels in the striatum of WT animals require 12-18 doses of $10 \mathrm{mg} \mathrm{kg}^{-1} \mathrm{PQ}$ (Prasad et al. 2009; Thiruchelvam et al. 2000), or combinations of PQ with MPTP or Maneb (Shepherd et al. 2006; Thiruchelvam et al. 2000). In our study, neither DA nor its metabolites change in the WT cohort after the $7^{\text {th }}$ dose. In contrast, the ApoD-KO mice show statistically significant PQ-dependent changes, revealing a lower amount of both DA and DOPAC (Fig. 1B). Our data suggest that the PQ regime used causes dopaminergic neurons to be impaired only in the ApoD-KO mice.

To test if these alterations are due to a higher dopaminergic cell death in ApoD-KO mice, we counted the number of TH-positive cells in the SN pars compacta $(\mathrm{SNc})$. As previously reported (McCormack et al. 2002), a decrease in the number of TH-positive neurons upon PQ treatment is evident, both in WT and ApoD-KO mice (Fig. 2A-B). However, no differential cell death is observed between the two genotypes. Also, no TUNEL-positive cells were observed following the chronic PQ treatment (not shown).

These results suggest that functional alterations in the PQ-vulnerable SNc neurons, instead of a higher rate of cell death, are the major consequence of the lack of ApoD when mice are exposed to our chronic PQ protocol, leading to the behavioral malfunction and the dopamine alterations observed. 


\section{Markers of glial reactivity and antioxidant response change in the substantia nigra in the}

\section{absence of ApoD.}

To further understand the functional consequences of ApoD absence in the nigrostriatal dopaminergic system, we evaluated the level of mRNA or protein expression of a set of genes (Fig. 3) in mesencephalic extracts including SNc obtained seven days after the last PQ dose. We focused on genes that can be expressed by glial cells, since ApoD is not expressed by nigral dopaminergic neurons (Ordonez et al. 2006). Damage to neurons, and oxidative damage in general, triggers mainly astrocyte responses (Bajo-Grañeras et al. 2011; Rossi and Volterra 2009) while inflammatory insults directly trigger microglial responses (Morale et al. 2006).

Since astroglial reactivity can be monitored by the expression of the cytoskeletal protein GFAP (Pekny and Nilsson 2005; Sofroniew 2009), we first evaluated the level of GFAP protein expression in ventral regions of the mesencephalon (Fig. S1). Our low-dose chronic PQ treatment causes an increase in basal astroglial reactivity and further increments over the already high basal level in ApoD-KO, as monitored by immunohistochemistry (Fig. 2B-C) and inmunoblot of SN protein extracts (Fig. 3A). These data suggest that ApoD is part of the mechanisms that restrain the extent of astrogliosis in vivo.

Heme oxygenase-1 (HO-1), a player in the early astroglial response and a sensitive and reliable reporter of tissue OS (Hsieh et al. 2010) still shows an increased up-regulation in ApoD-KO, both under control conditions and after being exposed to chronic PQ (Fig. 3B). In addition to its antioxidant direct functions, HO-1 triggers the expression of other protecting genes such as superoxide dismutase 2 (Sod2) and glial-derived neurotrophic factor (Gdnf) (Frankel et al. 2000; Hung et al. 2010). ApoD-KO mice display both a higher basal level and an enhanced response to 
chronic PQ treatment of these genes (Fig. 3C-D). It is especially noticeable the increase in Gdnf mRNA expression (Fig. 3D), considered as an endogenous protective mechanism particularly effective in the nigrostriatal system during Parkinson's disease (Morale et al. 2006; Villadiego et al. 2005). In contrast, 12/15 lipoxygenase (Alox15), an important mediator of neuronal cell death upon oxidative insult (Pallast et al. 2009), is down-regulated in the substantia nigra of ApoD-KO mice under control conditions, but its expression is up-regulated in response to PQ (Fig. 3E). The inducible NO synthase (Nos2), responsible for the production of NO and the subsequent generation of peroxynitrite, appears specifically down-regulated by chronic PQ in the ApoD-KO mice (Fig. 3F). Finally, the transcription of ApoE, an apolipoprotein with known antioxidant function (Poirier 2005), does not show genotype-dependent changes in the SN (not shown).

The molecular responses in the SN, together with the DA data described above, add to our previous findings (Ganfornina et al. 2008; Ganfornina et al. 2010) where the absence of ApoD provokes basal alterations in nervous system tissue homeostasis, generating an injury-like proinflammatory and pro-oxidant environment. Complex compensatory mechanisms are put forward, but they do not seem to include other nervous system apolipoproteins.

Since inflammation also plays a role in PQ toxicity (Mangano and Hayley 2009), we measured the transcript levels of Il6 and Tnfa, cytokines of the early response to PQ, and found no differential expression by genotype or chronic PQ treatment in the SN (not shown). Recently, we have found that acute high doses of PQ induce ApoD-dependent oligodendrocyte gene expression responses in the cerebellum (Bajo-Grañeras et al. 2011). In contrast, no genotype-dependent differential expression of myelin genes was seen in the SN in response to chronic PQ (not shown), indicating that this experimental paradigm is able to trigger a specific astroglial response to oxidative damage, with minor contribution of microglial or oligodendrocyte responses. However, 
since many of the genes we have studied so far in the SN are also expressed by nigral neurons, oligodendrocytes or microglia under pro-oxidant conditions, we need to study astrocytes isolated in culture in order to discern how much of the ApoD-dependent response observed upon OS is of astroglial nature.

\section{ApoD is part of the early response of astrocytes to oxidative stress.}

To test if astrocytes are a source of ApoD in a brain exposed to PQ we first used the human astroglioma cell line 1321N1 (Ortmann and Perkins 1977) and assayed ApoD expression at the mRNA (Fig. 4A) and protein (Fig. 4B-C) levels upon exposure to PQ.

We first assayed how ApoD mRNA levels change with time, from the moment of plating until confluence is reached in the culture dish (48h later) (Fig. S3A). As described for fibroblast-like and human astroglioma U373MG cell lines (Do Carmo et al. 2007), ApoD expression in 1321N1 cells is low when they are actively dividing, and is induced by growth arrest. PQ exposure was therefore performed in exponentially growing cells, to avoid the potential interaction of two different stimuli (growth arrest and OS), and for a maximum of 24 hours. We chose $500 \mu \mathrm{M}$ as the PQ dose reaching a maximum cell death (estimated from PI incorporation; not shown).

The time course of mRNA induction upon PQ exposure (Fig. 4A) indicates that ApoD is part of the early response of astrocytes to OS, reaching a peak induction $6 \mathrm{~h}$ after exposure to PQ. ApoD protein maintains its presence for longer periods (Fig. 4B-C and Fig. S3B). This is in agreement with the results obtained in vivo after chronic PQ treatment (Fig. S2). U373MG astroglioma cells show similar patterns of ApoD mRNA and protein expression (not shown). The decrease of ApoD transcript upon long exposures to PQ indicates the existence of a fine regulation of its expression, and that a continuous accumulation of ApoD protein might not be necessary or convenient for the 
cell after an oxidative insult. Interestingly, other acute-response genes in response to OS also show this finely timed regulation (Olesen et al. 2008; Wang et al. 2008).

\section{The stress responsive JNK signaling pathway regulates ApoD transcription in astrocytes.}

The Jun-N-terminal Kinase (JNK) signaling pathway is activated by PQ in PC12 and SH-SY5Y neuronal cell lines and to mediate PQ-induced dopaminergic cell apoptosis (Fei et al. 2008; Klintworth et al. 2007). Since Neural Lazarillo (NLaz), one of the ApoD homologous genes in Drosophila, is a downstream target of JNK in response to stress (Hull-Thompson et al. 2009), we hypothesized that the induction of ApoD transcript observed in astrocyte cell lines is triggered by JNK activation.

To test this idea we used the specific JNK inhibitor SP600125. As expected for targets of the JNK signaling cascade, both protein and mRNA levels of ApoD were reduced in the presence of the inhibitor in $1321 \mathrm{~N} 1$ cells treated with PQ (Fig. 4D,F). Furthermore, inhibition of JNK pathway activity in untreated control cultures reduces ApoD protein expression (Fig. 4E), indicating that JNK activity contributes to the basal level of ApoD expression. This effect also agrees with the observation that JNK inhibition in the presence of PQ leads to levels of mRNA below the control condition (Fig. 4F).

\section{ApoD deficient astrocytes become vulnerable to oxidative stress.}

To explore the functional significance of ApoD expression in astrocytes, we used primary astrocyte-enriched cortical glial cultures (referred to as astrocyte cultures henceforth) derived from postnatal brains (McCarthy and de Vellis 1980) of WT and ApoD-KO mice. 
Like human ApoD in astrocytoma cell lines, mouse ApoD in primary astrocytes is transcriptionally upregulated downstream of JNK signaling activity upon PQ exposure (Fig. 5A).

Since astrocytes are resistant to many forms of stress (Liddell et al. 2010) we tested whether ApoD is one of the factors contributing, through an autocrine mechanism, to their outstanding ability to survive. We assayed viability using the MTT assay. The lack of ApoD clearly renders primary astrocytes more vulnerable to PQ (Fig. 5B), and this effect is independent of the serum concentration used in the culture medium.

Astrocytes are known to undergo apoptosis when challenged with strong pro-inflammatory stimuli (Hu and Van Eldik 1996; Takuma et al. 2004). However, apoptotic cell death was negligible in astrocytoma cell lines upon PQ treatment (measured by active caspase 3 detection or Annexin V labeling; not shown). Likewise, cell death induced by PQ in primary astrocytes was mainly non-apoptotic, as evidenced by Annexin V-PI in vivo labeling and flow cytometry (Fig 5C). However, a clear difference between ApoD-KO and WT astrocytes is that a significant proportion of ApoD-KO astrocytes enter apoptosis, as revealed by Annexin V-positive PI-negative labeling (Fig. 5C, arrow). Similar results are obtained when apoptosis is assayed by TUNEL and quantified by fluorescence image densitometry (Fig. S4).

\section{ApoD modulates astrocyte reactivity.}

The increase in GFAP immunoreactivity in the SN of ApoD-KO mice (Fig. 2B-C and Fig. 3A), could be due to a higher number of reactive astrocytes in the tissue and/or a higher reactive state of astrocytes. A significant increase in GFAP immunoreactivity is also observed after 6 hours of PQ treatment in ApoD-KO astrocytes (Fig. 5D, lower right panel; see also immunoblot in Fig. 9E). This up-regulation of GFAP protein could be controlled at transcriptional levels, since the 
lack of ApoD up-regulates Nfla, a known activator of GFAP gene transcription, upon acute PQ treatment in the cerebellum (Bajo-Grañeras et al. 2011).

Following PQ treatment, WT astrocytes show a GFAP distribution in long cytoskeletal stress fibers commonly observed in reactive astrocytes (Pekny and Nilsson 2005; Sofroniew 2009). Many ApoD-KO astrocytes presented a spotted distribution of GFAP (Fig. 5D lower panels). Since the intermediary filament cytoskeleton is a sensitive sensor of toxic effects upon astrocytes (Pekny and Nilsson 2005; Renau-Piqueras et al. 1989), this cellular distribution of GFAP might be the result of a basal stress produced by the lack of ApoD.

\section{ApoD deficiency increases lipid peroxides in astrocytes without major apparent effects on earlier steps of the PQ-triggered oxidative cascade.}

Null mutants of ApoD or its homologous genes show an increased amount of peroxidated lipids in whole body fly extracts (Hull-Thompson et al. 2009; Sanchez et al. 2006) or in mouse brain homogenates (Ganfornina et al. 2008). Since lipid peroxidation in cellular membranes is a major sink for reactive oxygen species (ROS), a greater amount of peroxidated lipids can be due to a higher rate of production or to a slower rate of removal/recycling of the damaged membranes. High peroxidation rates would be accompanied by elevated ROS levels in the cell. We assayed aconitase activity as a very sensitive and early sensor of ROS levels in the cell, since its activity is quickly lost by oxidation-mediated loss of Fe from its Fe-S prosthetic group (Yan et al. 1997).

We find no decrease in aconitase activity in ApoD-KO astrocytes, and the activity reduction after a $2.5 \mathrm{~h}$ exposure to PQ was comparable in both genotypes (Fig. 6A). Therefore, the absence of ApoD does not directly increase the production or net level of ROS, since this would be evidenced by a stronger aconitase inactivation. However, ApoD-KO astrocytes show elevated 
basal levels of peroxidated lipids and proportionally higher levels of these ROS by-products in response to PQ exposure (Fig 6B,C).

These data strongly suggest that ApoD prevents the accumulation of peroxidated lipids in astrocytes, possibly by promoting their removal from damaged membranes.

\section{Astrocytes transcriptional response to oxidative stress is modified by ApoD.}

The results above indicate that, in the absence of $\mathrm{ApoD}$, astrocytes are still able to control the early steps in ROS management while accumulating lipid peroxides. In order to understand the global response of astrocytes to OS and the contribution of ApoD, we surveyed the transcription of 84 OS responding genes.

Thirty four genes do not show significant treatment or genotype-dependent changes. They are either genes not expressed by glial cultures, many of them in agreement with previous transcriptional profile analyses in astrocytes (Nakagawa and Schwartz 2004; Olesen et al. 2008), or genes that do not respond to the particular OS conditions we explore (500 $\mu \mathrm{M}$ PQ for $24 \mathrm{~h}$ ).

A set of 37 genes showed significant PQ-dependent changes in WT cells, with $31 \%$ of them being up-regulated (Table S1). This transcriptional profile reveals an interesting response of astrocytes to OS, as genes with pro-oxidant functions are down-regulated by PQ as part of an adaptive response to the oxidative insult. Moreover, many of the acute-response genes appear down-regulated at $24 \mathrm{~h}$ after their peak induction.

Only 8 genes show genotype-dependent changes in control conditions (Figure 7A). Five genes are up-regulated in ApoD-KO astrocytes, and in most of them (75\%) the changes mimic the response of WT cells under PQ treatment. 
ApoD-KO astrocytes respond to PQ with transcriptional changes in 46 genes. Eighteen genes (Table 1) display significantly different responses to PQ (more than two-fold difference in expression) between genotypes. A heat map representation is shown in Fig. 7B, and Fig. S6 displays a visual representation integrated with the functional networks formed among them. This pattern suggests that the absence of ApoD dampens the response to PQ of astrocytes, which are otherwise basally stressed (note that six out of the 18 genes also show genotype-dependent changes in basal conditions; boxed in Fig. 7).

The low number of genotype-affected genes indicates that changes in the response to PQ are not an indirect consequence of a pro-oxidant environment caused by the lack of ApoD, since that would trigger a generalized anti-oxidant defense response. Among the genes with a decreased response to PQ in the absence of ApoD are crucial ROS managing enzymes (Sod2, Sod3, Gpx3, Duox1 and Srxn1) and key proteins involved in inflammation signaling (Ptgs2-COX2, Ptgs1COX1, Il19).

In summary, the specific transcriptional changes observed can explain a higher vulnerability of ApoD-KO astrocytes to OS, and support that ApoD exerts autocrine protective functions.

\section{Exogenous addition of ApoD improves ApoD-KO astrocytes viability upon PQ exposure.}

Because the astrocyte response to PQ includes a JNK-mediated induction of ApoD (Fig. 5A), and without ApoD they become more vulnerable to OS (Fig. 5B), we hypothesized that addition of ApoD to ApoD-KO astrocytes would be beneficial. We simultaneously treated primary astrocytes with PQ and human ApoD (hApoD, purified from breast cyst fluid) at different concentrations (Fig. 8). Viability, measured by MTT assay, clearly improves when hApoD is added to ApoD-KO astrocytes (Fig. 8A). 
The effect reaches a plateau at 4-8 $\mathrm{nM}$, with additional increases of hApoD (up to $20 \mathrm{nM}$ ) resulting in no further viability improvement (not shown). Curiously, adding hApoD to WT astrocytes did not improve viability (Fig. 8B), and no significant changes were observed at high concentrations (up to $20 \mathrm{nM}$, not shown).

These results show that the ApoD available in the extracellular environment is recruited to the defense response organized by astrocytes against OS.

\section{Exogenous ApoD is internalized by astrocytes in a genotype-dependent manner.}

Exogenously administered ApoD has been described to be internalized by various cell lines and located in different subcellular compartments, including the nucleus and the cytoplasm (Do Carmo et al. 2007; Liu et al. 2001; Sarjeant et al. 2003; Thomas et al. 2003). The protective effect of hApoD reported in PQ-challenged astrocytes led us to test the internalization of hApoD by primary murine astrocytes, and whether there are differences between ApoD-KO and WT astrocytes.

Human ApoD was detected inside the cells when added to primary astrocytes, both by immunocytochemistry (Fig. 9A-C) and immunoblot of cell protein extracts after extensive replacement of media supernatant (Fig. 9D-E). The antibody used in these experiments fails to recognize the endogenous mouse ApoD in WT astrocytes (immunocytochemistry, not shown; lanes 1 and 3 in Fig. 9D,E). Internalization of hApoD was observed in ApoD-KO and WT astrocytes (Fig. 9B) with a lighter labeling in WT cells. Quantification of hApoD inmunoblot signals (Fig. 9D,E; red bars) confirmed the latter observation. Thus, ApoD-deficient mouse astrocytes do incorporate more hApoD than WT astrocytes. Remarkably, this difference in internalization is observed in control conditions, but not upon PQ treatment, where cells show a 
lower content of hApoD (Fig. 9D,E) after 24h treatment with PQ. This effect might be due to less incorporation or a faster transit of the exogenous protein through the cell.

The analysis of confocal images (Fig. 9C) show hApoD signal in a pattern resembling the intracellular membranous and vesicular compartments, particularly in the perinuclear area, but was not observed inside the nucleus, either in control conditions (Fig. 9C) or under PQ treatment (not shown).

To test whether this internalization might be a general and unspecific phenomenon, we performed the same experiment in two different cell types, HEK and HeLa cells that have negligible endogenous hApoD expression. When $\mathrm{hApoD}$ is added in the same range of concentrations used in the primary astrocyte experiments, HeLa, but not HEK cells, clearly internalize hApoD (Fig. S5). These experiments suggest that internalization is not due to unspecific endocytosis of proteins from the culture medium, and are compatible with a specific receptor-mediated endocytosis.

Changes in endocytosis and in the amount of intermediary cytoskeletal filaments have been shown to coexist in astrocytes exposed to pro-oxidant stimuli such as unconjugated bilirubin (Silva et al. 2001). Since ApoD influences astrocyte reactivity (Fig. 5D), we tested whether this effect was correlated with the amount of hApoD detected in cell extracts. We found that hApoD immunoreactivity was negatively correlated with GFAP (Fig. 9D,E, green bars). Therefore, the lack of ApoD is associated with more GFAP, particularly under PQ treatment both in vitro and in vivo (Fig. 9E; see also Fig. 2C-D and Fig. 5D), and the exogenous addition of hApoD is able to partially counteract this effect. Our data suggest that ApoD has an inhibitory effect on astrocyte reactivity that might be functionally linked to a finely regulated autocrine safety mechanism and, ultimately, to the protection of highly vulnerable dopaminergic neurons. 


\section{Discussion}

ApoD is linked to aging, degeneration and injury of the nervous system. Recent work from model organisms as divergent as plants, flies, and mice (Charron et al. 2008; Ganfornina et al. 2008; Hull-Thompson et al. 2009; Ruiz et al. 2011; Sanchez et al. 2006) has demonstrated that ApoD contributes to conserved survival mechanisms against OS. The link we previously found between lipid peroxides management in the brain and ApoD expression (Ganfornina et al. 2008) suggests that ApoD performs a protective function through the control of OS by-products. However, no direct proof was available for establishing a causal relationship between ApoD and the vulnerability of a functional nervous system to OS.

In this work we demonstrate that: i) ApoD contributes to the protection of the OS-sensitive dopaminergic system; ii) ApoD expression is triggered in astrocytes downstream of the stresssensitive JNK pathway; iii) ApoD contributes to restrain astrogliosis; and iv) ApoD secreted by astrocytes provides autocrine protection for these resilient glial cells against PQ-induced OS.

\section{ApoD function in the physiology of the nigrostriatal dopaminergic system}

Our results show that ApoD deficiency enhances the damaging effects of PQ in the mouse dopaminergic system. We chose a PQ treatment that avoids systemic toxicity and maximizes the OS effects on sensitive brain regions (Prasad et al. 2009). The evident bradikynesia of ApoD-KO mice, even under a mild PQ paradigm, reflects an indispensable role of ApoD for establishing a proper antioxidant defense in the brain. Functional alterations of dopaminergic systems are also supported by the significant differences in DA content found in the PQ-challenged brain of ApoDKO mice, despite diluting the striatal enrichment in PQ-sensitive dopaminergic terminals by including regions that are more resistant to PQ-induced OS (Wang et al. 2009). The basal increase 
in DA in the anterior brain of ApoD-KO mice is a puzzling observation that awaits further study. It might be due to compensatory increases in different dopaminergic regions. Interestingly, Chadchankar et al. (2011) show increased extracellular DA levels in the striatum of alphasynuclein deficient mice, indicating that compensatory mechanisms within the nigrostriatal system are taking place in different experimental approximations to PD. Moreover, the number of THpositive neurons show a trend to increase in the ApoD-KO SNc in control condition (Fig. 2B), which might explain, if confirmed with larger samples, the slight increase in forebrain DA levels.

Our study of the SN molecular response to PQ revealed both constitutive and OS-induced differences between ApoD-KO and WT animals. The expression changes comprise basally elevated levels of the glial reactivity sensor GFAP, also observed in situ in mesencephalic slices, and the antioxidant proteins HO-1 and Sod2, all of which stay elevated after a chronic PQ treatment. We also found specific PQ-dependent up-regulations for the OS-protecting factor Gdnf and the OS amplifier Alox15. Altogether these gene expression differences suggest the existence of a sustained OS in the neuronal environment of ApoD-KO mice, and an anomalous response of the gene network that needs to be organized to cope with the PQ-induced OS.

\section{ApoD role in the astroglial response to oxidative stress}

The lack of ApoD makes astrocytes more vulnerable to PQ treatment, and the exogenous addition of hApoD improves the viability of ApoD-deficient astrocytes. Since ApoD is expressed by astrocytes, we can conclude that it mediates an autocrine protection that in turn contributes to the nervous system homeostatic response to OS.

Loss-of-function mutants of ApoD or its homologues consistently show an increase in their basal levels of lipid peroxidation (Ganfornina et al. 2008; Hull-Thompson et al. 2009; Sanchez et 
al. 2006). Here we demonstrate that astrocytes accumulate more lipid peroxides if deprived of ApoD. A parsimonious hypothesis would predict that ApoD is a general antioxidant; OS would occur in its absence, and damage to lipids, proteins and DNA would appear as a consequence. Direct antioxidant properties have been reported in vitro for a recombinant form of ApoD, able to scavenge hydroxyl radicals and prevent DNA oxidation (Zhang et al. 2010). However, our data support that, in vivo, ApoD acts on specific components of the antioxidant defense tools of astrocytes. Its absence does not produce a generalized response. Elements of the antioxidant cascade like catalase, peroxiredoxins, thioredoxin reductases and most glutathione peroxidases do not have genotype-dependent changes of expression. With two superoxide dismutase genes (Sod2 and Sod3) up-regulated in ApoD-KO astrocytes in control conditions, superoxide radicals produced by metabolic activity are expected to be efficiently converted to $\mathrm{H}_{2} \mathrm{O}_{2}$. Detoxification of $\mathrm{H}_{2} \mathrm{O}_{2}$ by Gpx and Cat can be eventually overloaded, and dangerous levels of the highly reactive hydroxyl radical would slowly accumulate. This is compatible with the observation that aconitase activity (particularly sensitive to superoxide anions) is equally reduced in WT and ApoD-KO astrocytes, whereas lipid peroxides increase in the absence of ApoD. The net result is that some defense mechanisms are attenuated and some pro-oxidant mechanisms are exacerbated in ApoDKO astrocytes, leading to a higher vulnerability of these cells to oxidation.

Particularly interesting are the changes observed in genes related to the inflammatory response to PQ (I119 or Ptgs2 as examples of genes up-regulated by PQ, and Ptgs 1 among the genes downregulated by PQ), that show a diminished response to PQ in the absence of ApoD. ApoD function could thus contribute to turn on a proper inflammatory glial reaction during the initial phase of the response against an OS situation. 
The transcriptional regulation of ApoD by the JNK pathway, particularly involved in PQinduced OS (Klintworth et al. 2007; Peng et al. 2004), also supports the specificity of the protective role of ApoD. The temporally biphasic regulation of ApoD mRNA (early up-regulation followed by down-regulation), the small accumulation of protein observed in the striatum after chronic PQ treatment, and the plateau in viability rescue obtained after exogenous addition of ApoD to PQ-challenged cultures, suggest that astrocytes have mechanisms to control an upper limit of ApoD expression and function.

The viability rescue in ApoD-KO astrocytes is partial, indicating that ApoD is one of several genes involved in the response to OS. The effect of hApoD supplementation reaches saturation, suggesting the existence of a receptor-mediated process. However, no clear demonstration has been documented of a specific cell membrane receptor for ApoD. The fact that hApoD has no effect on the viability of PQ-challenged WT astrocytes suggests that cells negatively regulate the availability of putative ApoD receptors. Thus, astrocytes expressing endogenous ApoD would not be receptive to further ApoD additions. This idea is in agreement with the tight transcriptional regulation described above.

Another consequence of our results is that ApoD clearly modulates astrocyte reactivity, both in primary glial cultures and in vivo, contributing to its inhibition or restrain. Interestingly, another Lipocalin known to be induced upon stress in the vertebrate nervous system, Lcn2, mediates astrocyte reactivity. Over-expressing or adding Len2 to astrocytes sensitizes them to cytotoxic stimuli and induces astrogliosis (Lee et al. 2009), while decreasing Lcn2 correlates with decreased astrogliosis (Zheng et al. 2009). Turning on and off glial reactivity can be therefore accomplished by the complementary actions of the two Lipocalins. In this scenario, our data suggest that ApoD could be an off signal for astroglial reactivity. 
On the other hand, ApoE is expressed by glia and known to down-regulate CNS proinflammatory genes (Lynch et al. 2001). ApoD and ApoE have been proposed to perform redundant functions because of their lipid-binding properties (Terrisse et al. 1999). However, ApoE expression levels are similar in the PQ-challenged WT and ApoD-KO primary cultures (not shown), as well as in the mesencephalon of mice exposed to chronic PQ treatment (see above). Also, the induction of ApoE by peripheral nerve injury is decreased in ApoD-KO nerves (Ganfornina et al. 2010), further supporting the hypothesis that these two lipoproteins play different and not compensatory functions. Lastly, and contrary to ApoD, ApoE has been recently shown to be induced by inhibiting the JNK pathway (Pocivavsek and Rebeck 2009).

We propose a role for ApoD in maintaining the glial response to OS and the concomitant inflammatory reaction under fixed limits. Our results suggest that ApoD, ApoE and Lcn2 form a complementary team controlling the on-off signals that tune the glial response to injury. Assessing the role of ApoD as on-off signal in the neuronal environment is next in our research program, by studying the position and contribution of ApoD in the functional network established among astrocytes, microglia and the OS vulnerable neurons. 


\section{Acknowledgements}

We thank J.R. Acebes for technical assistance, and the Lazarillo Lab (M. Ruiz, N. García-Mateo, M. del Caño \& A. Pérez-Castellanos) for their helpful discussions and positive criticisms. We thank C. Sánchez-Vicente at the Confocal Microscopy Service in IBGM for technical assistance. Cell lines 1321N1, U373, HEK, and HeLa were kindly provided by M. L. Nieto (IBGM-CSIC, Valladolid, Spain) and F. Aguado (Univ. Barcelona, Spain). Purified hApoD was a gift from E. Rassart (Univ. Quebec á Montreal, Canada). Work supported by grants to M. D.G. and D.S. (MEC grant BFU2005-00522; JCyL grant VA049A05; and MICINN grant BFU2008-01170). R.B-G. was supported by a JCyL fellowship (GRS/278/A/08). 


\section{References}

Bajo-Grañeras R, Sanchez D, Gutierrez G, Gonzalez C, Carmo SD, Rassart E, Ganfornina MD. 2011. Apolipoprotein $D$ alters the early transcriptional response to oxidative stress in the adult cerebellum. $\mathrm{J}$ Neurochem In press.

Chadchankar H, Ihalainen J, Tanila H, Yavich L. 2011. Decreased reuptake of dopamine in the dorsal striatum in the absence of alpha-synuclein. Brain Res 1382:37-44.

Charron JB, Ouellet F, Houde M, Sarhan F. 2008. The plant Apolipoprotein D ortholog protects Arabidopsis against oxidative stress. BMC Plant Biol 8:86.

de Magalhaes JP, Curado J, Church GM. 2009. Meta-analysis of age-related gene expression profiles identifies common signatures of aging. Bioinformatics 25(7):875-881.

Do Carmo S, Levros Jr L-C, Rassart E. 2007. Modulation of apolipoprotein D expression and translocation under specific stress conditions. Biochimica et Biophysica Acta (BBA) - Molecular Cell Research 1773(6):954-969.

Fei Q, McCormack AL, Di Monte DA, Ethell DW. 2008. Paraquat neurotoxicity is mediated by a Bak-dependent mechanism. J Biol Chem 283(6):3357-64.

Frankel D, Mehindate K, Schipper HM. 2000. Role of heme oxygenase-1 in the regulation of manganese superoxide dismutase gene expression in oxidatively-challenged astroglia. Journal of Cellular Physiology 185(1):80-86.

Ganfornina MD, Do Carmo S, Lora JM, Torres-Schumann S, Vogel M, Allhorn M, González C, Bastiani MJ, Rassart E, Sanchez D. 2008. Apolipoprotein D is involved in the mechanisms regulating protection from oxidative stress. Aging Cell 7(4):506515.

Ganfornina MD, Do Carmo S, Martínez E, Tolivia J, Navarro A, Rassart E, Sanchez D. 2010. ApoD, a glia-derived apolipoprotein, is required for peripheral nerve functional integrity and a timely response to injury. Glia 58(11):1320-1334.

Hsieh H-L, Wang H-H, Wu C-Y, Yang C-M. 2010. Reactive Oxygen Species-Dependent cFos/Activator Protein 1 Induction Upregulates Heme Oxygenase-1 Expression by Bradykinin in Brain Astrocytes. Antioxidants \& Redox Signaling 0(0).

Hu J, Van Eldik LJ. 1996. S100 beta induces apoptotic cell death in cultured astrocytes via a nitric oxide-dependent pathway. Biochimica Biophysica Acta 1313(239 -245).

Hull-Thompson J, Muffat J, Sanchez D, Walker DW, Benzer S, Ganfornina MD, Jasper H. 2009. Control of Metabolic Homeostasis by Stress Signaling Is Mediated by the Lipocalin NLaz. PLoS Genet 5(4):e1000460. 
Hung S-Y, Liou H-C, Fu W-M. 2010. The mechanism of heme oxygenase-1 action involved in the enhancement of neurotrophic factor expression. Neuropharmacology 58(2):321-329.

Klintworth H, Newhouse K, Li T, Choi W-S, Faigle R, Xia Z. 2007. Activation of c-Jun NTerminal Protein Kinase Is a Common Mechanism Underlying Paraquat- and Rotenone-Induced Dopaminergic Cell Apoptosis. Toxicological Sciences 97(1):149162.

Lee S, Park J-Y, Lee W-H, Kim H, Park H-C, Mori K, Suk K. 2009. Lipocalin-2 Is an Autocrine Mediator of Reactive Astrocytosis. J Neurosci 29(1):234-249.

Liddell JR, Robinson SR, Dringen R, Bishop GM. 2010. Astrocytes retain their antioxidant capacity into advanced old age. Glia 58(12):1500-1509.

Liu Z, Chang G-Q, Leibowitz SF. 2001. Apolipoprotein D interacts with the long form leptin receptor: a hypothalamic function in the control of energy homeostasis. The FASEB Journal.

Loerch PM, Lu T, Dakin KA, Vann JM, Isaacs A, Geula C, Wang J, Pan Y, Gabuzda DH, Li $C$ and others. 2008. Evolution of the aging brain transcriptome and synaptic regulation. PLoS ONE 3(10):e3329.

Lynch JR, Morgan D, Mance J, Matthew WD, Laskowitz DT. 2001. Apolipoprotein E modulates glial activation and the endogenous central nervous system inflammatory response. J Neuroimmunol 114(1-2):107-13.

Mangano EN, Hayley S. 2009. Inflammatory priming of the substantia nigra influences the impact of later paraquat exposure: Neuroimmune sensitization of neurodegeneration. Neurobiol Aging 30(9):1361-78.

McCarthy KD, de Vellis J. 1980. Preparation of separate astroglial and oligodendroglial cell cultures from rat cerebral tissue. The Journal of Cell Biology 85(3):890-902.

McCormack AL, Thiruchelvam M, Manning-Bog AB, Thiffault C, Langston JW, CorySlechta DA, Di Monte DA. 2002. Environmental Risk Factors and Parkinson's Disease: Selective Degeneration of Nigral Dopaminergic Neurons Caused by the Herbicide Paraquat. Neurobiology of Disease 10(2):119-127.

Miller RL, James-Kracke M, Sun GY, Sun AY. 2009. Oxidative and inflammatory pathways in Parkinson's disease. Neurochem Res 34(1):55-65.

Morale MC, Serra PA, L'Episcopo F, Tirolo C, Caniglia S, Testa N, Gennuso F, Giaquinta G, Rocchitta G, Desole MS and others. 2006. Estrogen, neuroinflammation and neuroprotection in Parkinson's disease: Glia dictates resistance versus vulnerability to neurodegeneration. Neuroscience 138(3):869-878. 
Nakagawa T, Schwartz JP. 2004. Gene expression profiles of reactive astrocytes in dopamine-depleted striatum. Brain Pathol 14(3):275-80.

Olesen BT, Clausen J, Vang O. 2008. Characterization of the transcriptional profile in primary astrocytes after oxidative stress induced by Paraquat. NeuroToxicology 29(1):13-21.

Ordonez C, Navarro A, Perez C, Astudillo A, Martinez E, Tolivia J. 2006. Apolipoprotein D expression in substantia nigra of Parkinson disease. Histol Histopathol 21(4):361-6.

Ortmann R, Perkins JP. 1977. Stimulation of adenosine 3' :5'-monophosphate formation by prostaglandins in human astrocytoma cells. Inhibition by nonsteroidal antiinflammatory agents. J Biol Chem 252(17):6018-25.

Pallast S, Arai K, Wang X, Lo EH, Van Leyen K. 2009. 12/15-Lipoxygenase targets neuronal mitochondria under oxidative stress. Journal of Neurochemistry 111(3):882-889.

Pekny M, Nilsson M. 2005. Astrocyte activation and reactive gliosis. Glia 50(4):427-434.

Peng J, Mao XO, Stevenson FF, Hsu M, Andersen JK. 2004. The herbicide paraquat induces dopaminergic nigral apoptosis through sustained activation of the JNK pathway. $J$ Biol Chem 279(31):32626-32.

Perea G, Navarrete M, Araque A. 2009. Tripartite synapses: astrocytes process and control synaptic information. Trends Neurosci 32(8):421-31.

Pocivavsek A, Rebeck GW. 2009. Inhibition of c-Jun N-terminal kinase increases apoE expression in vitro and in vivo. Biochem Biophys Res Commun 387(3):516-20.

Poirier J. 2005. Apolipoprotein E, cholesterol transport and synthesis in sporadic Alzheimer's disease. Neurobiol Aging 26(3):355-61.

Prasad K, Tarasewicz E, Mathew J, Strickland PAO, Buckley B, Richardson JR, Richfield EK. 2009. Toxicokinetics and toxicodynamics of paraquat accumulation in mouse brain. Experimental Neurology 215(2):358-367.

Prasad K, Winnik B, Thiruchelvam M, Buckley B, Mirochnitchenko O, Richfield E. 2007. Prolonged toxicokinetics and toxicodynamics of paraquat in mouse brain. Environ Health Perspect 115(10):1448-53.

Renau-Piqueras J, Zaragoza R, De Paz P, Baguena-Cervellera R, Megias L, Guerri C. 1989. Effects of prolonged ethanol exposure on the glial fibrillary acidic protein-containing intermediate filaments of astrocytes in primary culture: a quantitative immunofluorescence and immunogold electron microscopic study. J Histochem Cytochem 37(2):229-240. 
Rossi D, Volterra A. 2009. Astrocytic dysfunction: insights on the role in neurodegeneration. Brain Res Bull 80(4-5):224-32.

Ruiz M, Sanchez D, Canal I, Acebes A, Ganfornina MD. 2011. Sex-dependent modulation of longevity by two Drosophila homologues of human Apolipoprotein D, GLaz and NLaz. Experimental Gerontology doi: DOI: 10.1016/j.exger.2011.02.014.

Sanchez D, Lopez-Arias B, Torroja L, Canal I, Wang X, Bastiani MJ, Ganfornina MD, Walker DW, Muffat J, Rundel C and others. 2006. Loss of glial lazarillo, a homolog of apolipoprotein $\mathrm{D}$, reduces lifespan and stress resistance in Drosophila. Curr Biol 16(7):680-6.

Sarjeant JM, Lawrie A, Kinnear C, Yablonsky S, Leung W, Massaeli H, Prichett W, Veinot JP, Rassart E, Rabinovitch M. 2003. Apolipoprotein D Inhibits Platelet-Derived Growth Factor-BB-Induced Vascular Smooth Muscle Cell Proliferated by Preventing Translocation of Phosphorylated Extracellular Signal Regulated Kinase 1/2 to the Nucleus. Arterioscler Thromb Vasc Biol 23(12):2172-2177.

Shepherd KR, Lee E-SY, Schmued L, Jiao Y, Ali SF, Oriaku ET, Lamango NS, Soliman KFA, Charlton CG. 2006. The potentiating effects of 1-methyl-4-phenyl-1,2,3,6tetrahydropyridine (MPTP) on paraquat-induced neurochemical and behavioral changes in mice. Pharmacology Biochemistry and Behavior 83(3):349-359.

Silva R, Mata L, Gulbenkian S, Brites D. 2001. Endocytosis in Rat Cultured Astrocytes Is Inhibited by Unconjugated Bilirubin. Neurochemical Research 26(7):793-800.

Sofroniew MV. 2009. Molecular dissection of reactive astrogliosis and glial scar formation. Trends in Neurosciences 32(12):638-647.

Song YJC, Halliday GM, Holton JL, Lashley T, O'Sullivan SnS, McCann H, Lees AJ, Ozawa T, Williams DR, Lockhart PJ and others. 2009. Degeneration in Different Parkinsonian Syndromes Relates to Astrocyte Type and Astrocyte Protein Expression. Journal of Neuropathology \& Experimental Neurology 68(10):1073-1083.

Takuma K, Baba A, Matsuda T. 2004. Astrocyte apoptosis: implications for neuroprotection. Progress in Neurobiology 72(111-127).

Terrisse L, Seguin D, Bertrand P, Poirier J, Milne R, Rassart E. 1999. Modulation of apolipoprotein $D$ and apolipoprotein $E$ expression in rat hippocampus after entorhinal cortex lesion. Brain Res Mol Brain Res 70(1):26-35.

Thiruchelvam M, Richfield EK, Baggs RB, Tank AW, Cory-Slechta DA. 2000. The Nigrostriatal Dopaminergic System as a Preferential Target of Repeated Exposures to Combined Paraquat and Maneb: Implications for Parkinson's Disease. J Neurosci 20(24):9207-9214. 
Thomas EA, George RC, Sutcliffe JG. 2003. Apolipoprotein D modulates arachidonic acid signaling in cultured cells: implications for psychiatric disorders. Prostaglandins, Leukotrienes and Essential Fatty Acids 69(6):421-427.

Van Dijk W, Do Carmo S, Rassart E, Dalhlback B, Sodetz J. 2006. The plasma Lipocalins $\alpha_{1}$-acid glycoprotein, apolipoprotein $D$, apolipoprotein $M$ and complement $C 8 \gamma$. In: Akerstrom B, Borregaard N, Flower D, Salier J, editors. Lipocalins. Georgetown, Texas: Landes Bioscience. p 140-66.

Villadiego J, Mendez-Ferrer S, Valdes-Sanchez T, Silos-Santiago I, Farinas I, Lopez-Barneo J, Toledo-Aral JJ. 2005. Selective Glial Cell Line-Derived Neurotrophic Factor Production in Adult Dopaminergic Carotid Body Cells In Situ and after Intrastriatal Transplantation. J Neurosci 25(16):4091-4098.

Walker DW, Muffat J, Rundel C, Benzer S. 2006. Overexpression of a Drosophila Homolog of Apolipoprotein D Leads to Increased Stress Resistance and Extended Lifespan. Current Biology 16(7):674-679.

Wang J, Duhart HM, Xu Z, Patterson TA, Newport GD, Ali SF. 2008. Comparison of the time courses of selective gene expression and dopaminergic depletion induced by MPP+ in MN9D cells. Neurochem Int 52(6):1037-43.

Wang X, Zaidi A, Pal R, Garrett AS, Braceras R, Chen XW, Michaelis ML, Michaelis EK. 2009. Genomic and biochemical approaches in the discovery of mechanisms for selective neuronal vulnerability to oxidative stress. BMC Neurosci 10:12.

Yan LJ, Levine RL, Sohal RS. 1997. Oxidative damage during aging targets mitochondrial aconitase. Proc Natl Acad Sci U S A 94(21):11168-72.

Zhang Y, Cong Y, Wang S, Zhang S. 2010. Antioxidant activities of recombinant amphioxus (Branchiostoma belcheri) apolipoprotein D. Molecular Biology Reports:1-5.

Zheng LT, Lee S, Yin GN, Mori K, Suk K. 2009. Down-regulation of lipocalin 2 contributes to chemoresistance in glioblastoma cells. Journal of Neurochemistry 111(5):12381251. 


\section{FIGURE AND TABLE LEGENDS}

Fig. 1. Lack of ApoD alters the nigrostriatal dopaminergic system and results in bradykinesia upon chronic PQ treatment. (A) Open field test of locomotor exploratory behavior performed 6 days after the $7^{\text {th }} \mathrm{PQ}$ injection $\left(10 \mathrm{mg} \mathrm{kg}^{-1}\right)$. While this mild chronic exposure to PQ does not alter locomotor output in WT controls, the velocity of movement, number of ambulatory events and time spent in stereotypic movements are significantly decreased in ApoD-KO mice. (B) The concentration of anterior brain DA and its metabolites (DOPAC and HVA) was determined by HPLC 7 days after the $7^{\text {th }}$ PQ injection. DA and DOPAC were reduced upon PQ treatment only in ApoD-KO mice. A genotype-dependent basal increase in DA was also observed. Data shown as mean $\pm \mathrm{SD} . \mathrm{N}=10$ mice/genotype. Unpaired Student's t-test; *: $p<0.05$.

Fig. 2. ApoD influences astrocyte reactivity levels upon chronic PQ treatment without alterations in nigrostriatal neuronal cell death. (A) Representative photomicrographs of TH immunohistochemistry in coronal mesencephalic slices performed 7 days after the $7^{\text {th }}$ PQ injection $\left(10 \mathrm{mg} \mathrm{kg}^{-1}\right)$. (B) The number of TH-positive neurons in the SNc region decrease in a PQdependent manner in both ApoD-KO and WT mice. No genotype-dependent differences are detected. (C) GFAP immunostaining in mesencephalic slices performed as in A. (D) Quantification of GFAP immunoreactivity in the substantia nigra and interpeduncular regions reveals basal differences in the level of astroglial reactivity, and an enhanced response to chronic PQ treatment in the ApoD-KO mice. Data shown as mean $\pm \mathrm{SD}(\mathrm{B}, \mathrm{D}) . \mathrm{N}=6$ mice/genotype. Unpaired Student's t-test; $*: p<0.05$. 
Fig. 3. Markers of astroglial reactivity and antioxidant response are altered in the substantia nigra of ApoD-KO mice. (A-C) Immunoblot analysis of OS-dependent genes (GFAP, HO-1 and SOD2) shows elevated basal and PQ-triggered expression in the ApoD-KO substantia nigra. Graphs represent mean \pm SD of 2-4 independent experiments (protein levels quantified by band densitometry normalized to $\beta$-actin signal). (D-F) Quantitative RT-PCR analysis of Gdnf, Alox15 and Nos 2 expression.. Protein and mRNA were extracted 7 days after the $7^{\text {th }}$ PQ injection. $N=10$ mice/genotype. Dashed lines represent: the average protein level obtained in sham-injected WT animals (A-C), or a twofold change in mRNA concentration with respect to the calibrator sample (D-E). Statistical differences assayed by unpaired Student's t-test (A-C) and by Mann-Whitney Utest (D-F).*: $p<0.05$.

Fig. 4. Human ApoD is induced by PQ downstream of JNK in the astroglioma cell line 1321N1. (A) ApoD mRNA expression is transiently induced upon exposure to $500 \mu \mathrm{M}$ PQ. Relative amounts with respect to untreated cells in each time point is shown. (B) High levels of ApoD protein expression are observed by densitometry analysis of the immunoreactivity signal after PQ treatment (24h). Calibration bar: $50 \mu \mathrm{m}$. (C) Quantification of ApoD by immunoblot analysis after PQ treatment (24h). (D) Activation of the JNK pathway is required for the PQ-dependent ApoD induction. ApoD is detected by immunoblot upon PQ exposure (24 h) plus increasing concentrations of the JNK inhibitor SP600125. (E) Effect of JNK inhibitor on ApoD protein expression in control conditions. (F) JNK inhibitor prevents the induction of ApoD mRNA upon PQ exposure (4 h). Quantitative RT-PCR values are represented with respect to control conditions (calibrator sample). Densitometry values in C-E are shown as percentage of control value after normalization to $\beta$-actin signal. Dashed lines represent: a twofold change in mRNA concentration 
with respect to calibrator sample (A), or the average protein level obtained in untreated cells (BE). Graphs in C-E represent mean \pm SD of 3-4 independent experiments. Statistical differences assayed by ANOVA (A), unpaired Student's t-test (B-E) and Mann-Whitney U-test (F).*: $p<$ 0.05 .

Fig. 5. Increased vulnerability and reactivity of ApoD-KO primary astrocytes. (A) JNK pathway activity is required for the PQ-dependent ApoD induction in primary astroglial cultures. Mouse ApoD mRNA expression upon exposure to $500 \mu \mathrm{M}$ PQ (6 h) with or without JNK inhibitor SP600125 $(20 \mu \mathrm{M})$. (B) Astrocyte viability measured by MTT assay upon $24 \mathrm{~h}$ PQ treatment. Percent survival calculated in relation to untreated cells of each genotype. Pictures are representatives of a single experiment. Graph shows mean \pm SD of 3-4 independent experiments in each serum condition. (C) Flow cytometry analysis of Annexin V-FITC and PI double-labeled cells. Most PQ induced cell death is non-apoptotic (upper-left quadrant) but a significantly higher proportion of cells enter apoptosis in ApoD-KO primary astrocytes cultures (arrow). Dot plots show a representative experiment. Graph shows mean \pm SD of 3 independent measures. (D) Astroglial reactivity assayed by GFAP expression in primary cultures. In addition to the qualitative differences in GFAP distribution, ApoD-KO astrocytes show a higher induction of GFAP upon PQ treatment (6 h). Statistical differences assayed by Mann-Whitney U-test (A) or Student's t-test (C-D).*: $p<0.05$. Calibration bars: $50 \mu \mathrm{m}$ (B), $10 \mu \mathrm{m}$ (D).

Fig. 6. Lipid peroxidation increases in ApoD-KO astrocytes without alterations in aconitase activity. (A) Reduction of aconitase activity (Bioxytech Aconitase-340 assay) after PQ treatment $(2.5 \mathrm{~h}, 500 \mu \mathrm{M})$ is similar in ApoD-KO and WT astrocytes. (B-C) Lipid peroxidation, assayed by 
TBARS (B) or by MDA-586 assay (C) is basally increased in ApoD-KO astrocytes. Exposure to PQ (24h) further increases the accumulation of lipid peroxide adducts (B). Data represent mean \pm SD of 2-4 independent experiments. Statistical differences assayed by unpaired Student's t-test, *: $p<0.05$.

Fig. 7. Quantitative RT-PCR expression profiles of primary astrocyte cultures. (A) Subset of genes with statistically significant changes in basal expression levels in ApoD-KO astrocytes. Expression level in WT untreated astrocytes is used as calibrator for each gene. (B) Cluster analysis and heat map of the 18 genes that showed genotype-dependent significant differences in their response to PQ (24 h treatment). Columns represent samples. Rows represent genes. Color coded relative quantification scaling is shown at the bottom. Fold change values for each gene are listed in Table 1 . Genes common to subset A and B are boxed. Only statistically supported changes (Mann-Whitney's U-test, $p<0.05$ ) with a fold change $\geq 2$ are shown.

Fig. 8. Exogenous addition of human ApoD (hApoD) promotes survival of astrocytes upon PQ exposure in the absence of endogenous ApoD. (A) Addition of increasing concentrations of hApoD to mouse astrocyte cultures at the time of PQ treatment improves survival up to 2.2 fold in ApoD-KO astrocytes. (B) Survival of WT astrocytes is however unaltered by hApoD treatment. Percent survival upon $24 \mathrm{~h}$ PQ treatment (MTT assay) relative to the untreated cells of each genotype is shown. Data presented as mean \pm SD of 3-5 independent experiments. Statistical differences assayed by ANOVA test, *: $p<0.05$. 
Fig. 9. Exogenous ApoD effects on survival are accompanied by internalization of the protein and modulation of astroglial reactivity. (A-C) Immunofluorescence analyses of hApoD incorporation. Nuclei are labeled with DAPI. (A) Fluorescence microscopy images of primary mouse astrocytes in control conditions in the absence (left panel) or presence of $4 \mathrm{nM} h A p o D$. (B) Confocal microscopy images show that hApoD is internalized by both ApoD-KO and WT astrocytes. Contours of cells were delineated after DIC optic images (not shown). (C) Maximal projection of a z-series. Orthogonal z-projections of the axes depicted by dashed lines are shown at the bottom and right side of the image. Human ApoD immunoreactivity is located in cytoplasmic vesicle-like structures, but not inside the nucleus. Calibration bars: $50 \mu \mathrm{m}$ (A), $10 \mu \mathrm{m}$ (B-C). (D-E) Immunoblot analysis of hApoD and GFAP content in cell extracts of WT (D) and ApoD-KO (E) primary astrocytes in control conditions and after PQ treatment $(24 \mathrm{~h})$ with or without addition of $4 \mathrm{nM}$ hApoD. Protein levels, quantified by band densitometry, are shown as percentage of control value after normalization to $\beta$-actin signal.

Table 1. Subset of genes with genotype-dependent expression in response to PQ treatment. Fold change (FC) with respect to untreated cultures of each genotype is listed (mean $\pm \mathrm{SD}$ of 4 replicas). Criteria for gene selection were: (i) Statistically supported changes with PQ (MannWhitney's U-test, $p<0.05)$, and (ii) $\mathrm{FC}(\mathrm{KO})= \pm 2 \cdot \mathrm{FC}(\mathrm{WT})$. 


\section{SUPPLEMENTARY FIGURE AND TABLE LEGENDS}

Fig. S1. Mesencephalic regions analyzed to evaluate SNc dopaminergic neuronal cell death and astroglial reactivity. Coronal slices $(200 \mu \mathrm{m})$ including the regions of interest were obtained from fresh tissue, fixed and processed as described in the Methods section. The areas outlined in dashed lines were selected in each slice to count the total number of TH-positive neuronal cell bodies. The areas outlined with boxes were selected in each slice for GFAP immunofluorescence quantification. $\mathrm{MG}$, medial geniculate; $\mathrm{SuC}$, superior colliculus; $\mathrm{CG}$, central gray; SNC, substantia nigra pars compacta; SNR, substantia nigra pars reticulata; VTA, ventral tegmental area; IP, interpeduncular nucleus.

Fig. S2. ApoD protein levels are increased after acute pro-inflammatory and pro-oxidant stimuli, and it is maintained at high levels upon chronic PQ treatment. (A) Immunoblot analysis of ApoD protein levels in striatum. Protein extracts were performed either after 5 weeks of PQ treatment ( $5 \mathrm{w}: 7$ days after the $7^{\text {th }}$ injection at $10 \mathrm{mg} \mathrm{kg}^{-1}$ ), or $12 \mathrm{~h}$ after a single injection of LPS $(3.3 \mathrm{mg}$ $\left.\mathrm{kg}^{-1}\right)$ or PQ $\left(30 \mathrm{mg} \mathrm{kg}^{-1}\right)$. No ApoD immunoreactivity is detected in the ApoD-KO mice. Protein levels were quantified by band densitometry normalized to $\beta$-actin signal.

Fig. S3. Time course of hApoD expression in astroglioma 1321N1 cells. (A) ApoD mRNA temporal expression profile in untreated cells measured by qRT-PCR. Time is measured as hours after plating. Confluence is reached at $48 \mathrm{~h}$. Relative amounts with respect to the $3 \mathrm{~h}$ time point (calibrator sample) are shown. (B) Time course of hApoD protein expression upon PQ exposure measured by immunoblot. Bar graphs show the mean \pm SD of 4 independent experiments. 
Densitometry values were normalized to $\beta$-actin and shown as percent of control (untreated cells) value.

Fig. S4. Apoptotic cell death is increased in ApoD-KO primary astrocytes. (A) Representative low magnification fluorescence images after TUNEL assay. Apoptotic nuclei are shown in pale green. Non-apoptotic nuclei are labeled in deep blue by DAPI. (B) Positive control performed in primary macrophages after treating cells with DNAseI. (C) Quantification of percentage of TUNELpositive cells in 10 optic fields (10x objective) per genotype and condition. Data represented as mean \pm SD. Statistical differences assayed by Student's t-test, ${ }^{*} p<0.05$.

Fig. S5. Internalization of hApoD into cells is not due unspecific endocytosis. (A) Immunoreactivity against hApoD is not detected in the HEK cell line after addition of $4 \mathrm{nM}$ hApoD for 24 h. (B) HeLa cells, however, do internalize hApoD after the same treatment.

Fig. S6. Gene pathway analysis of the ApoD-dependent subsets of anti-oxidant responsive genes performed with Gene Network Central Pro ${ }^{\mathrm{TM}}$ (http://gncpro.sabiosciences.com). (A-B) Relationships among genes showing differential response to PQ treatment between WT and ApoD-KO astrocytes. (C) Relationships among genes with significantly different basal levels of expression in ApoD-KO mice. Circles represent genes whose expression has been measured in our qRT-PCR array. Diamonds represent genes with regulatory or physical interactions with the assayed genes. 
Table S1. Gene expression changes to PQ treatment in WT primary astrocyte cultures. Fold change (FC) with respect to untreated cultures is listed (mean $\pm \mathrm{SD}$ of 4 replicas). All cases are statistically supported (Mann-Whitney's U-test, $p<0.05$ ) and have a FC $>2$. 
Figure 1
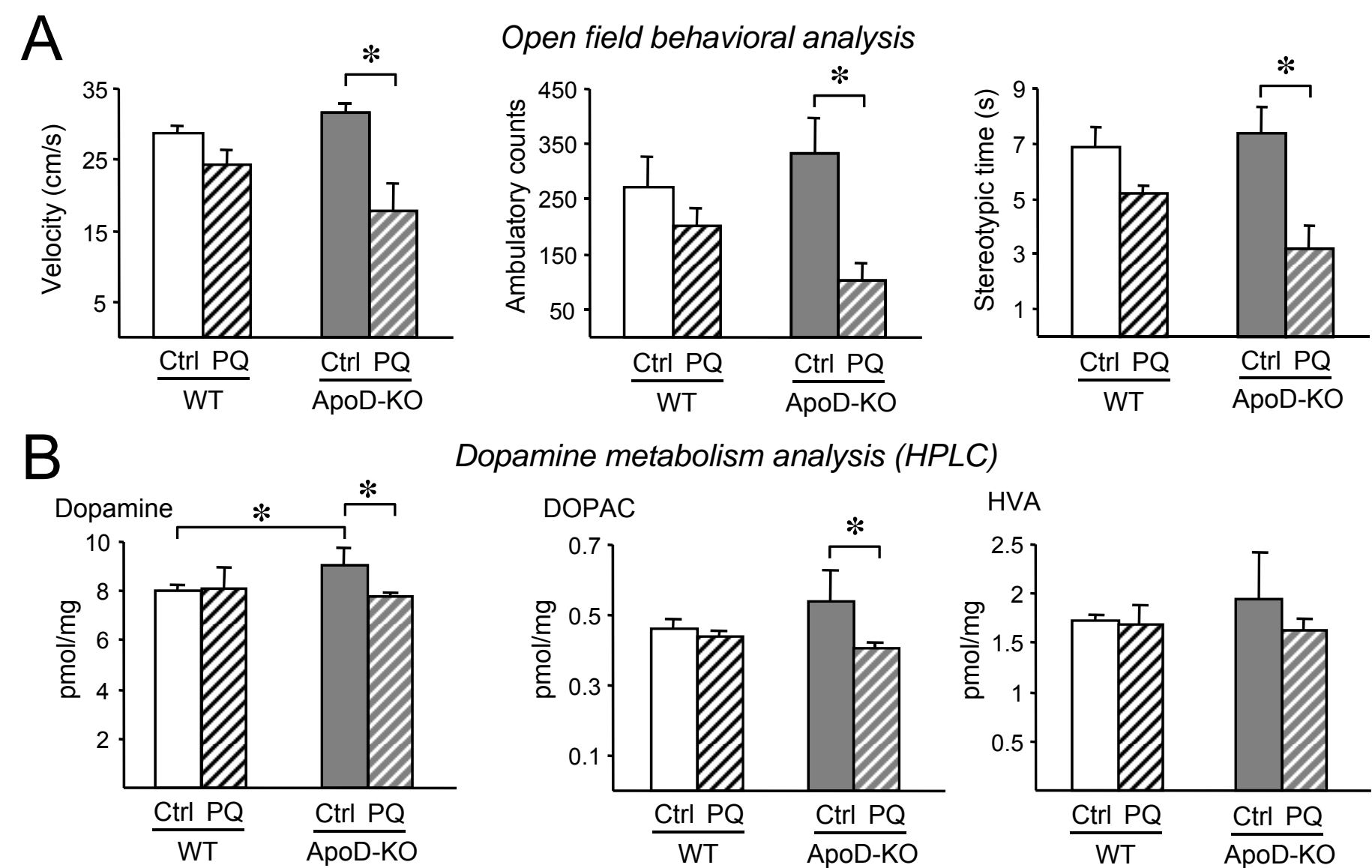

Dopamine metabolism analysis (HPLC)
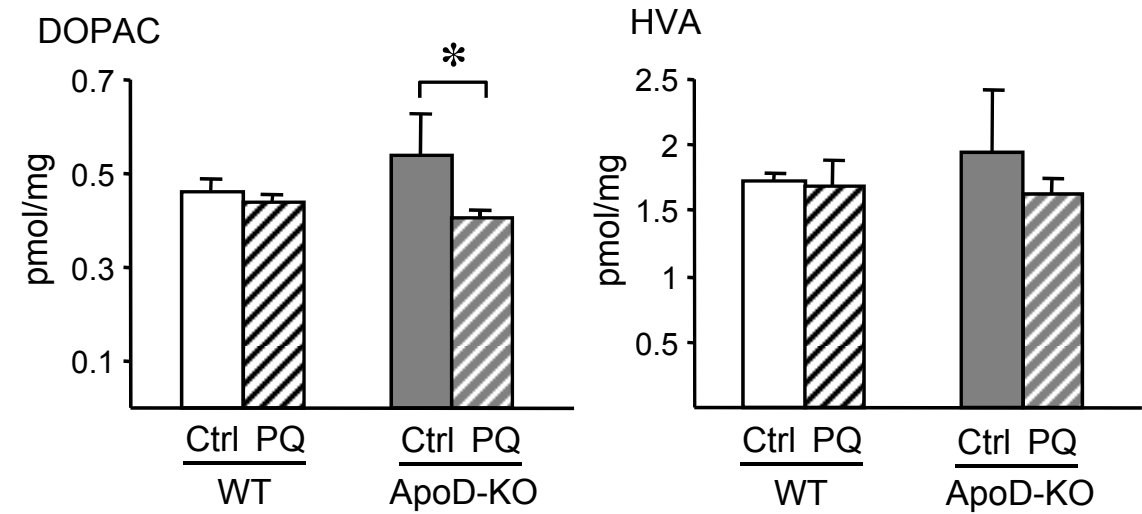
Figure 2
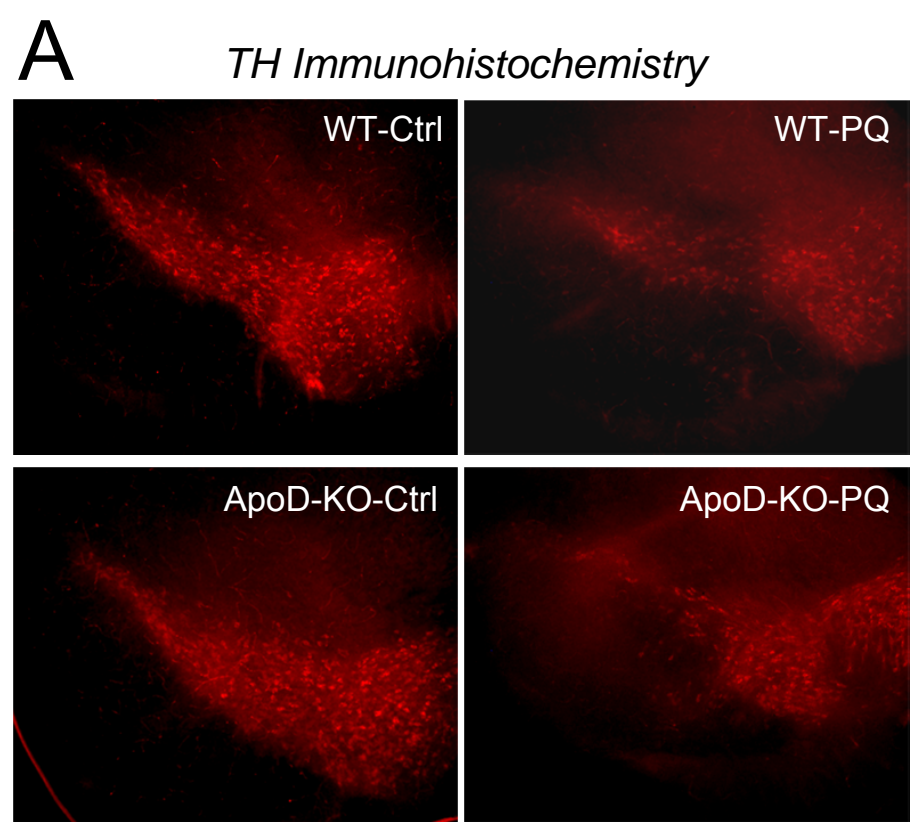

B

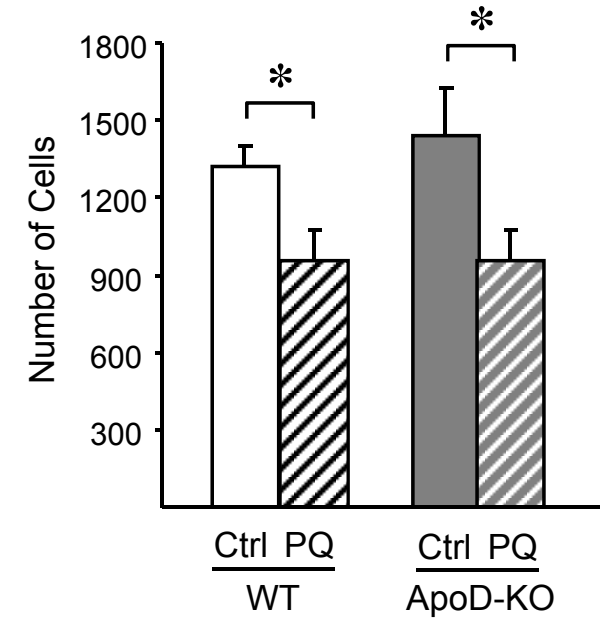

C GFAP Immunohistochemistry

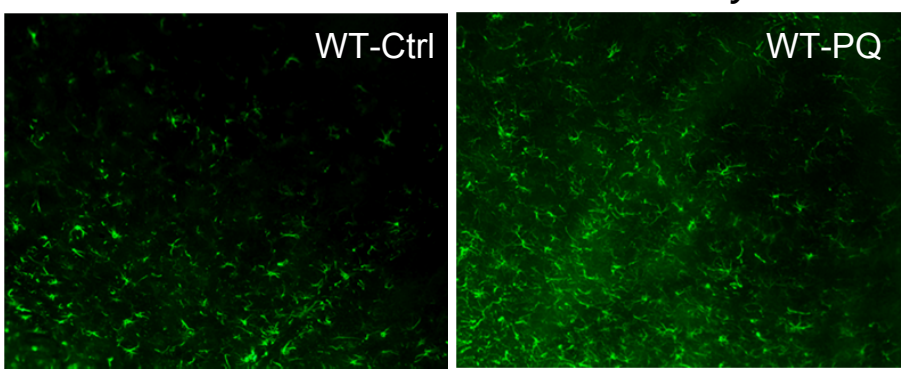

D
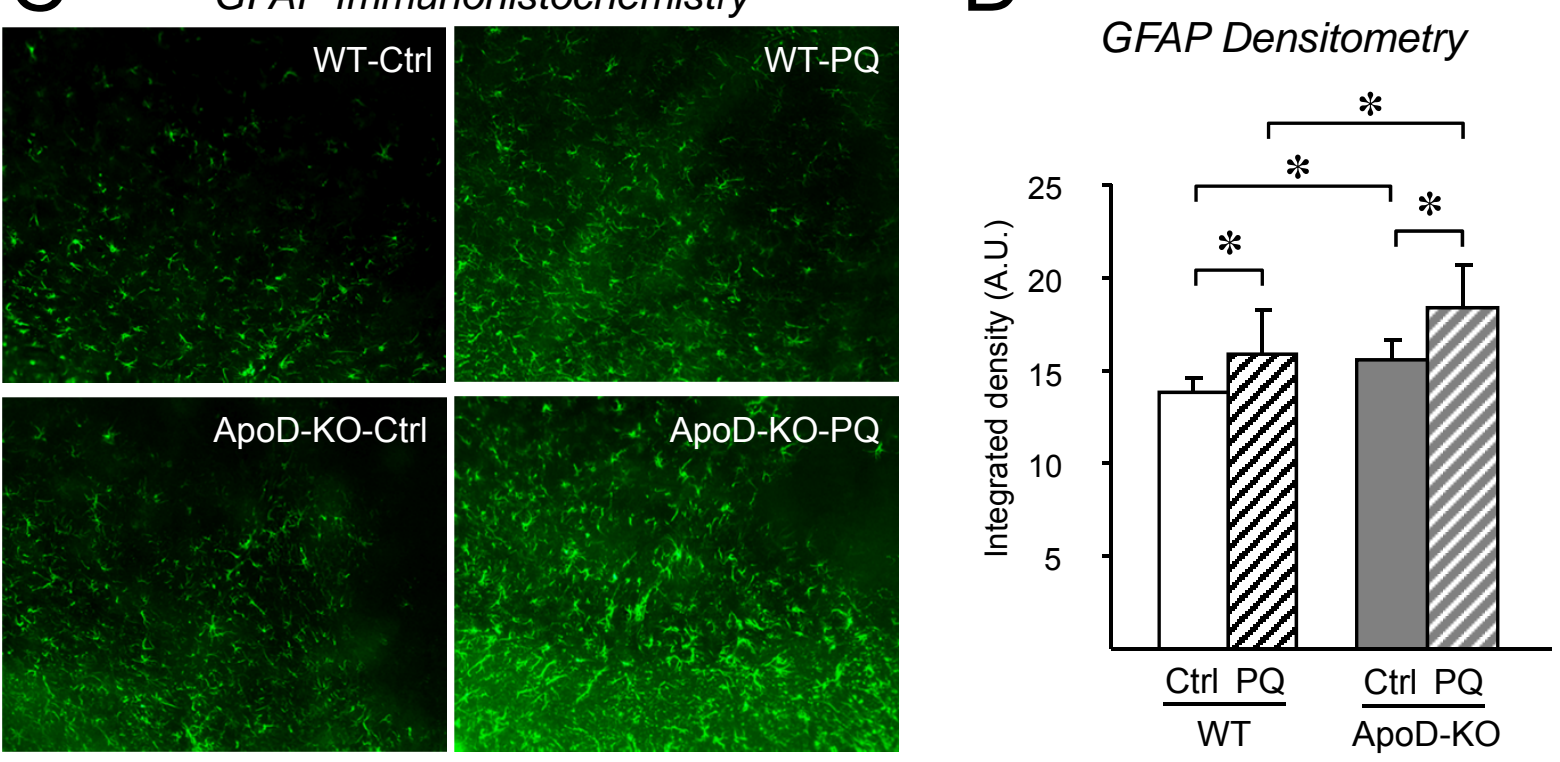
Figure 3
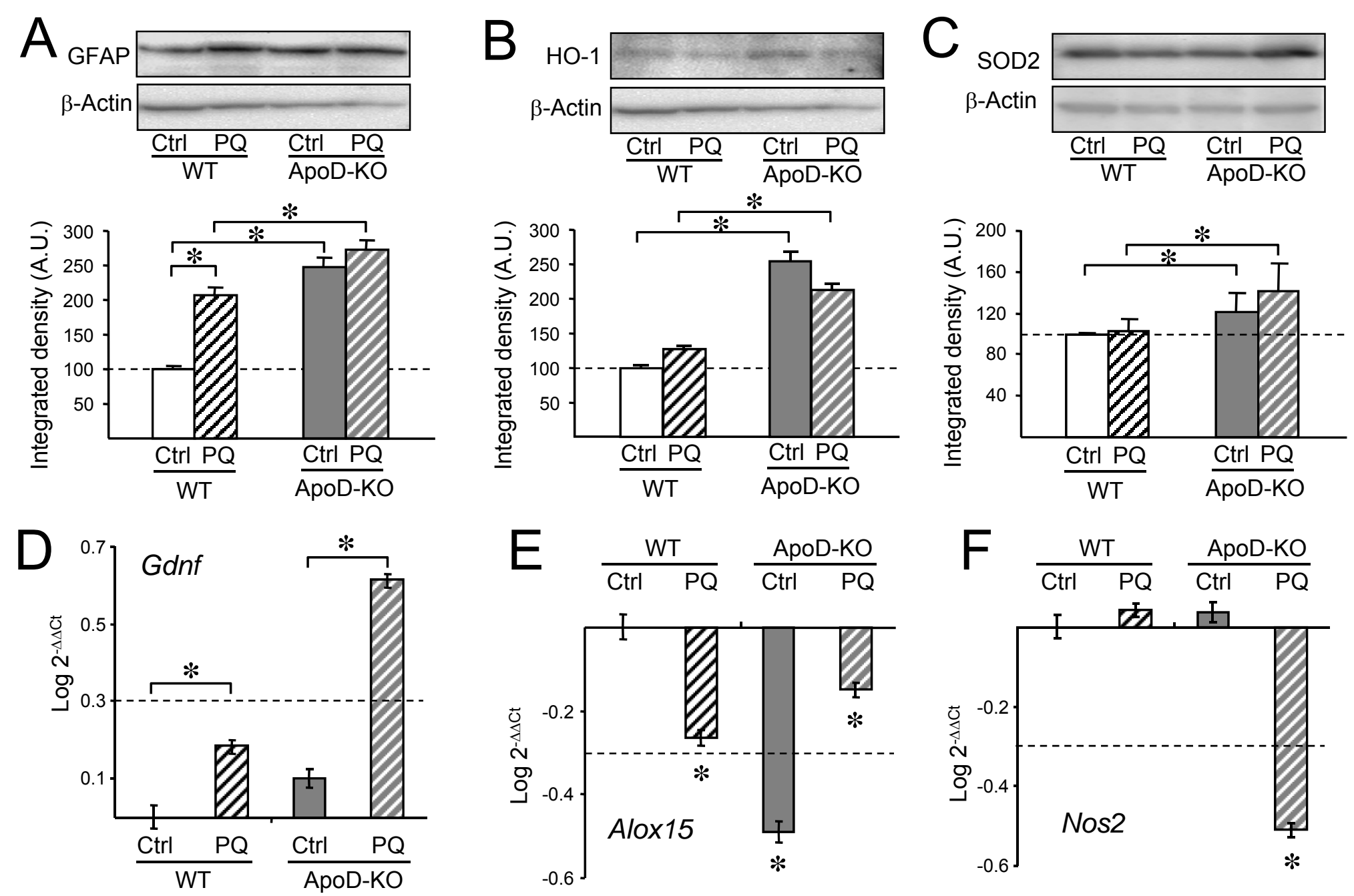


\section{Figure 4}

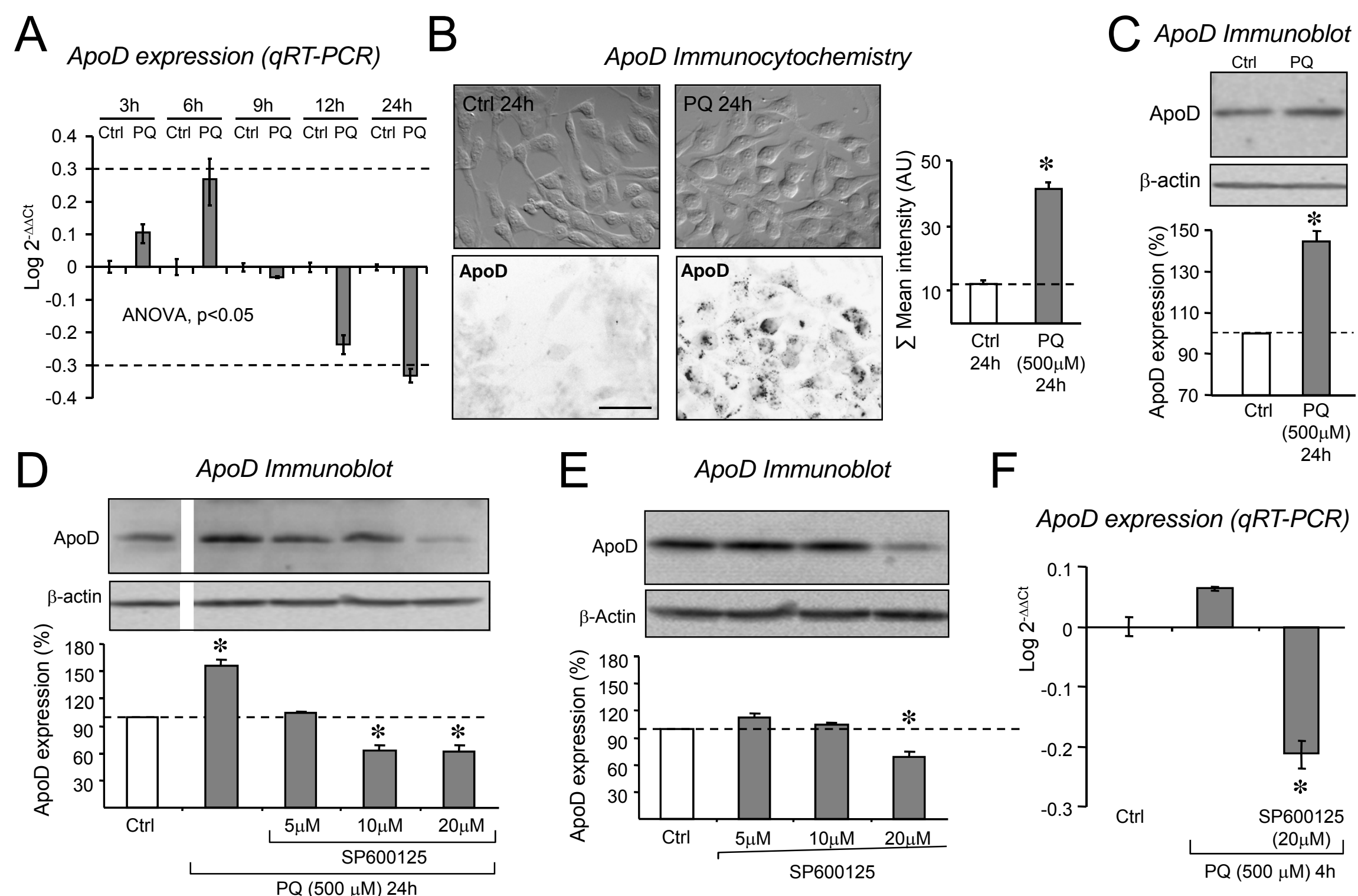


Figure 5 A ApoD expression B
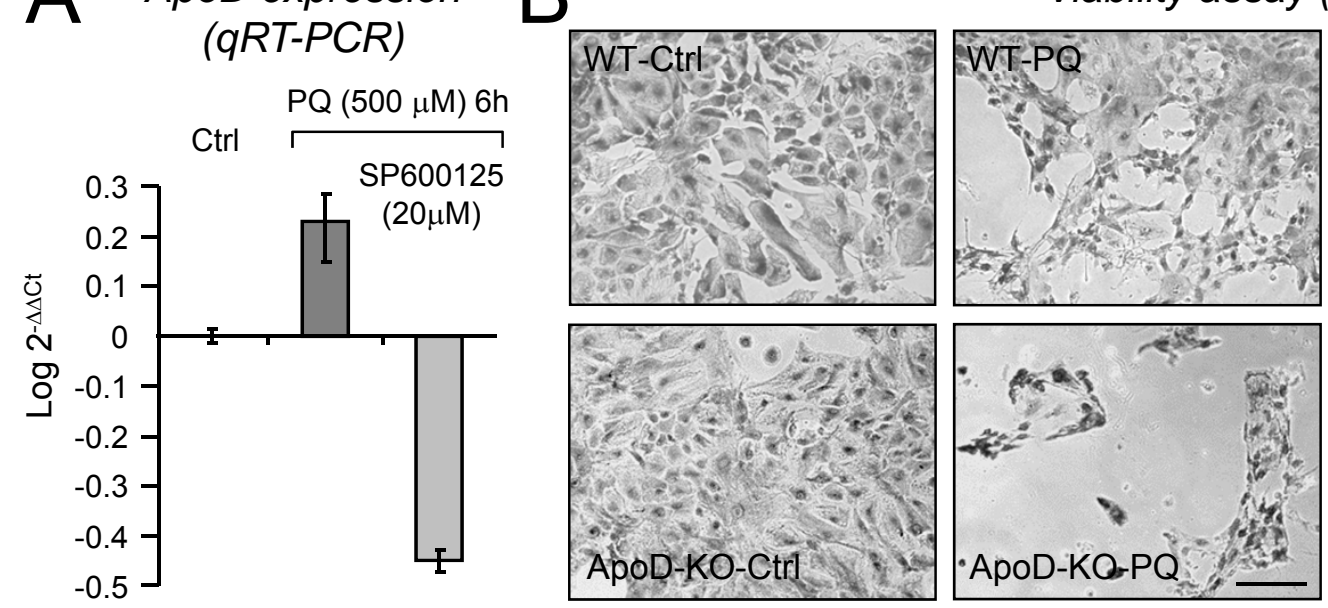

Viability assay (MTT)

C

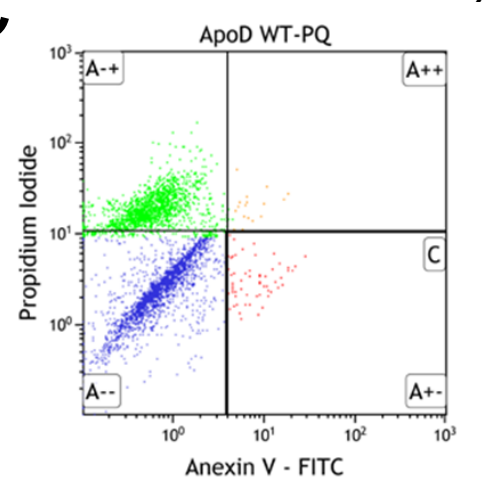

Apoptosis assay (Annexin $V-P I)$
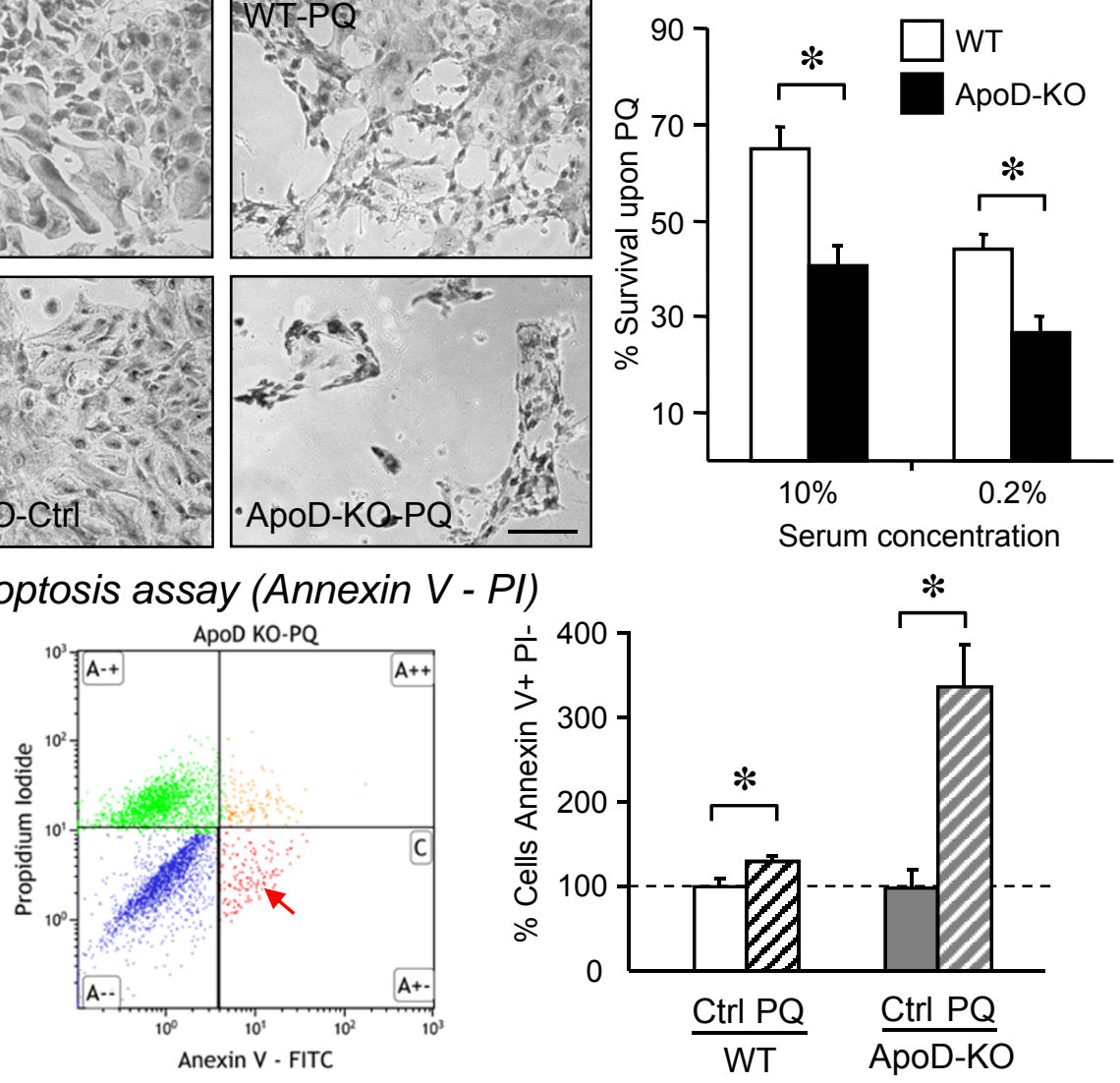

D GFAP Immunofluorescence

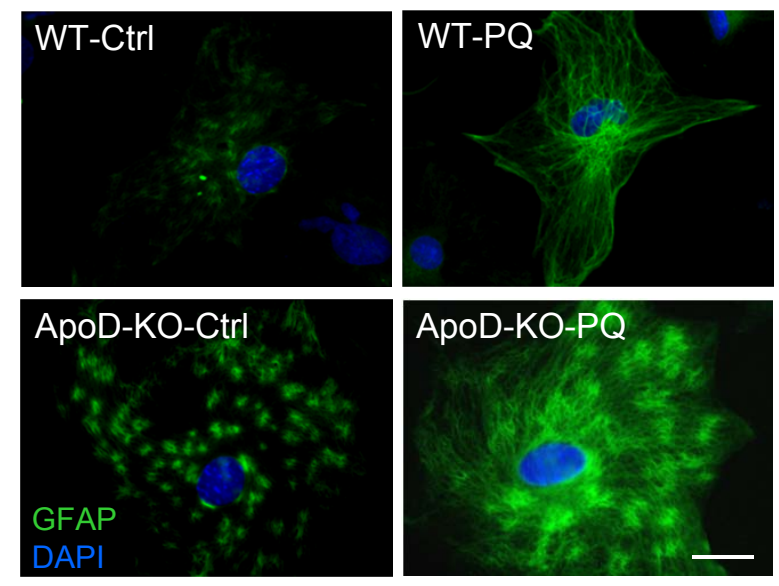

GFAP densitometry

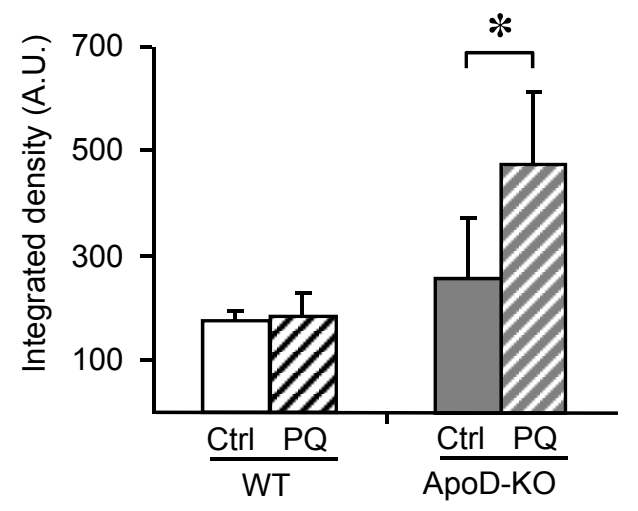


Figure 6

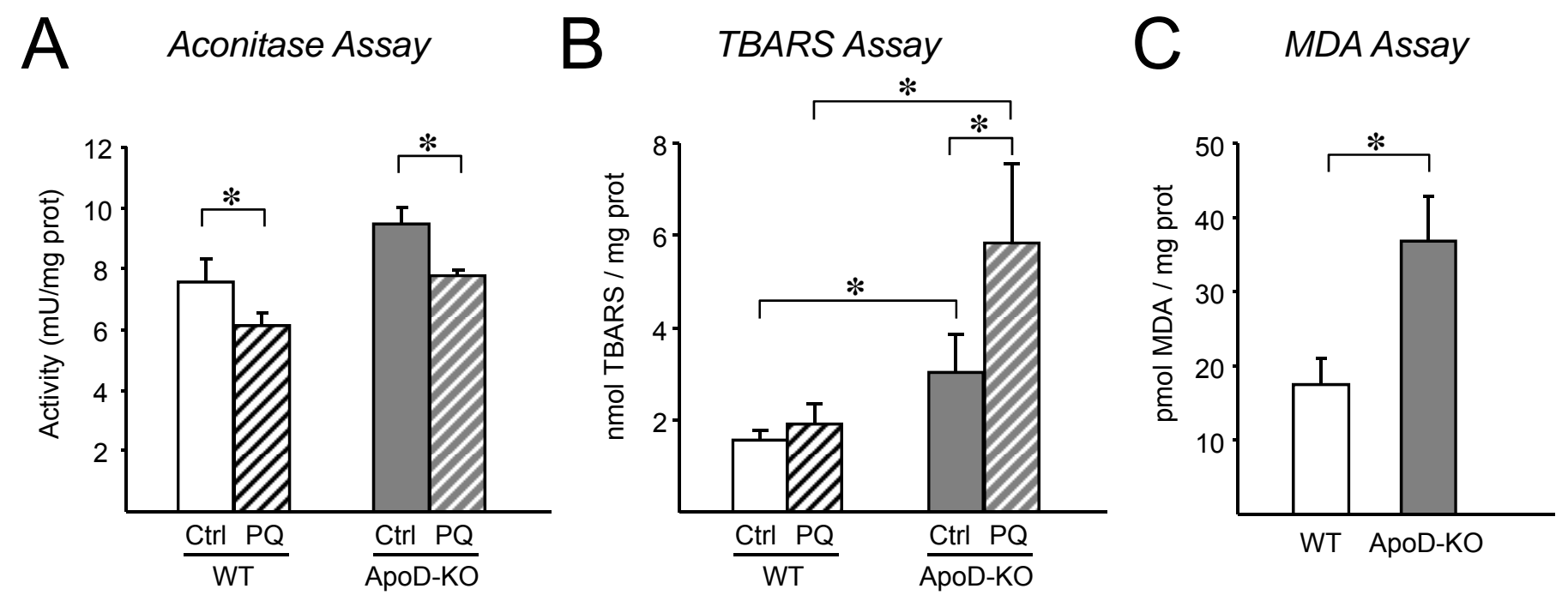


Figure 7

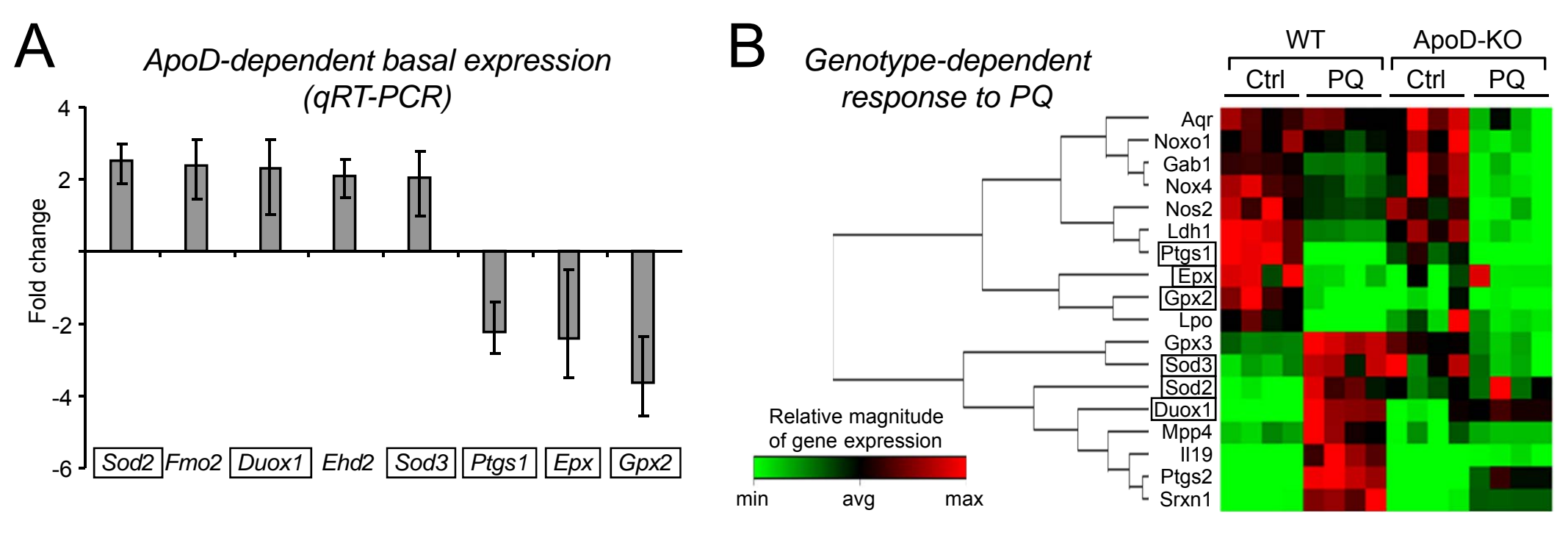


Figure 8

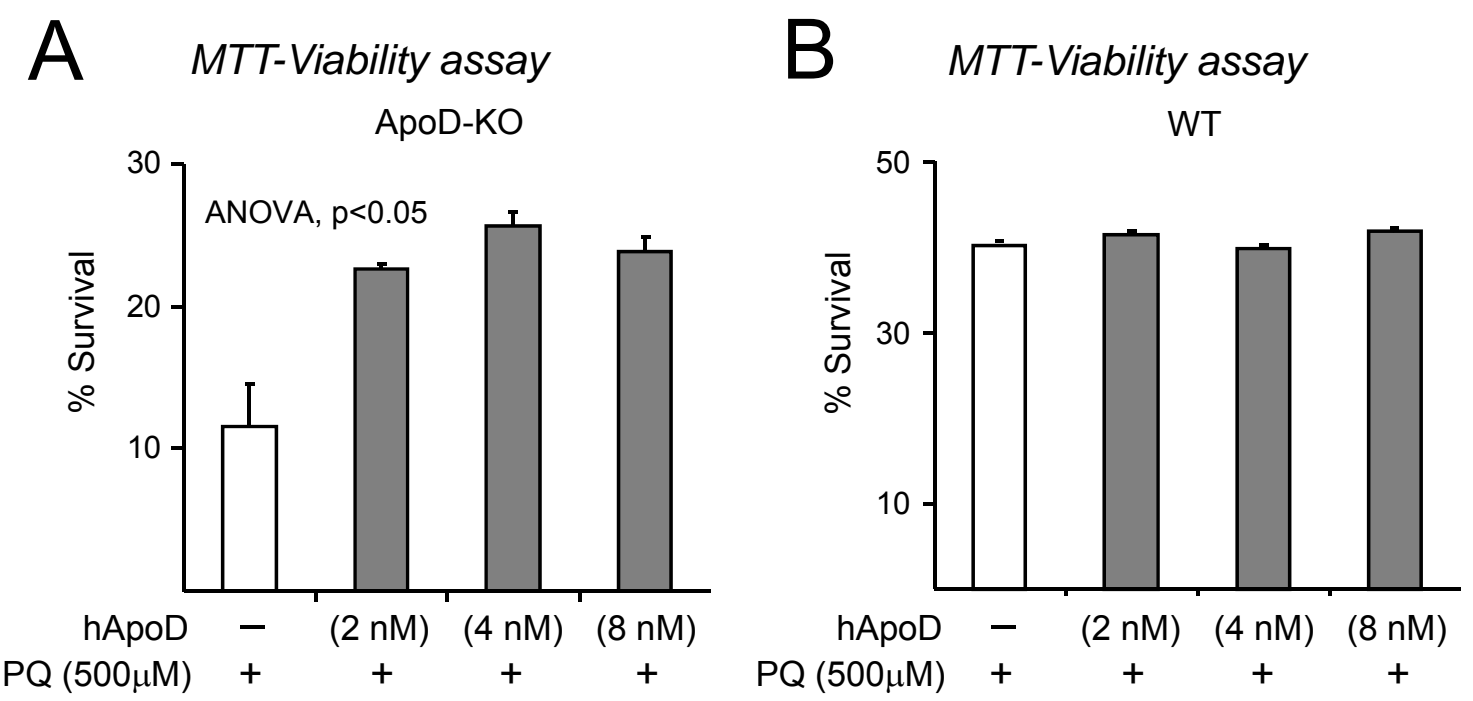


Figure 9
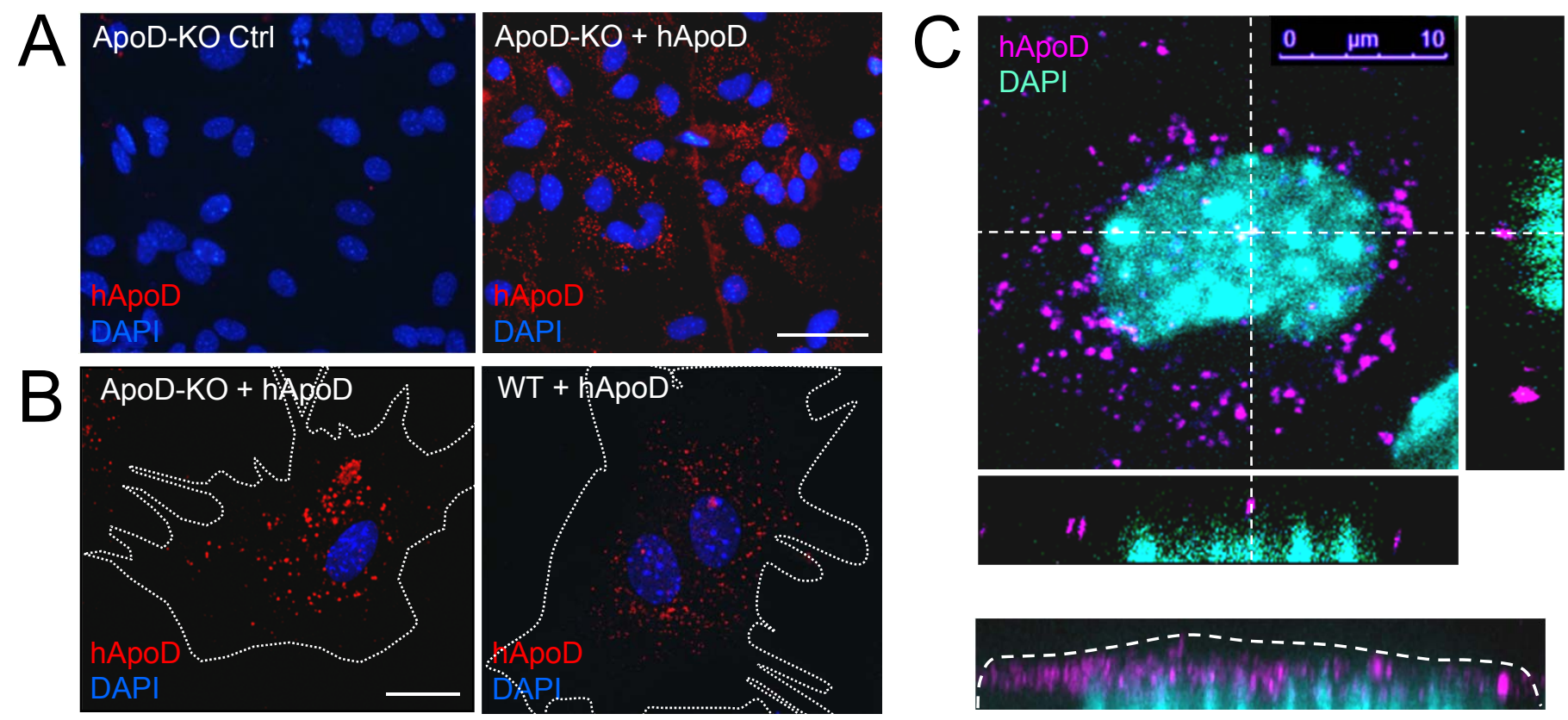

D Immunoblot (WT culture)
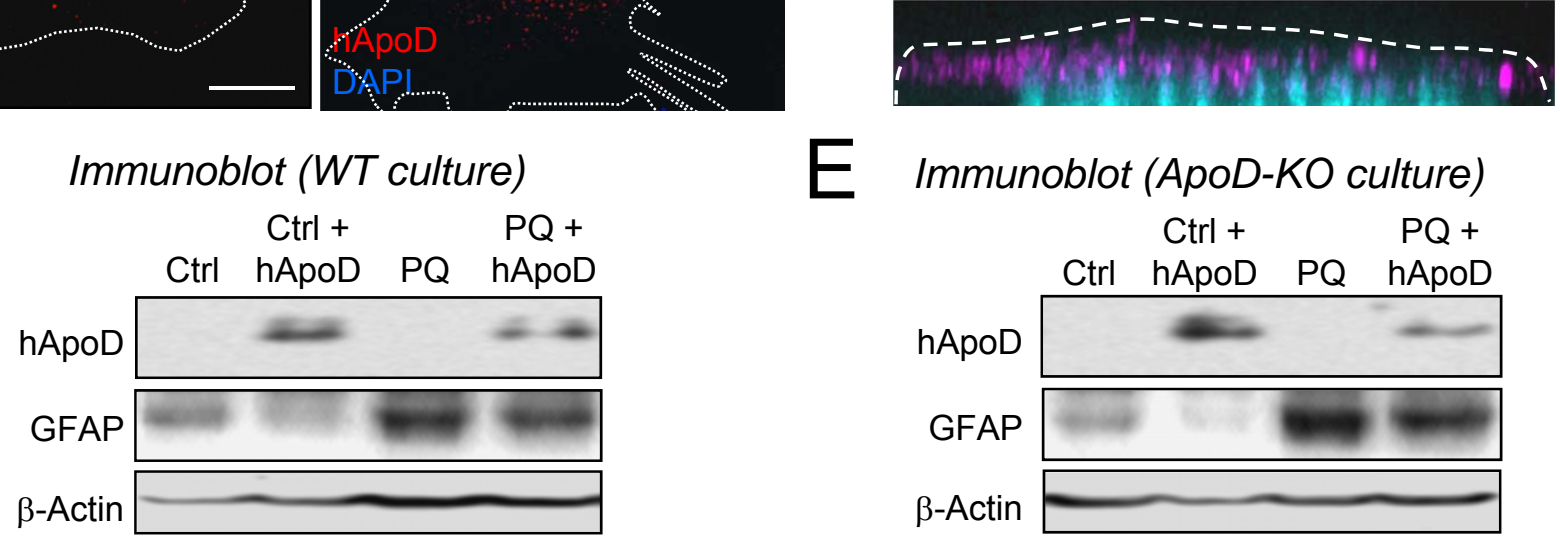

E Immunoblot (ApoD-KO culture)
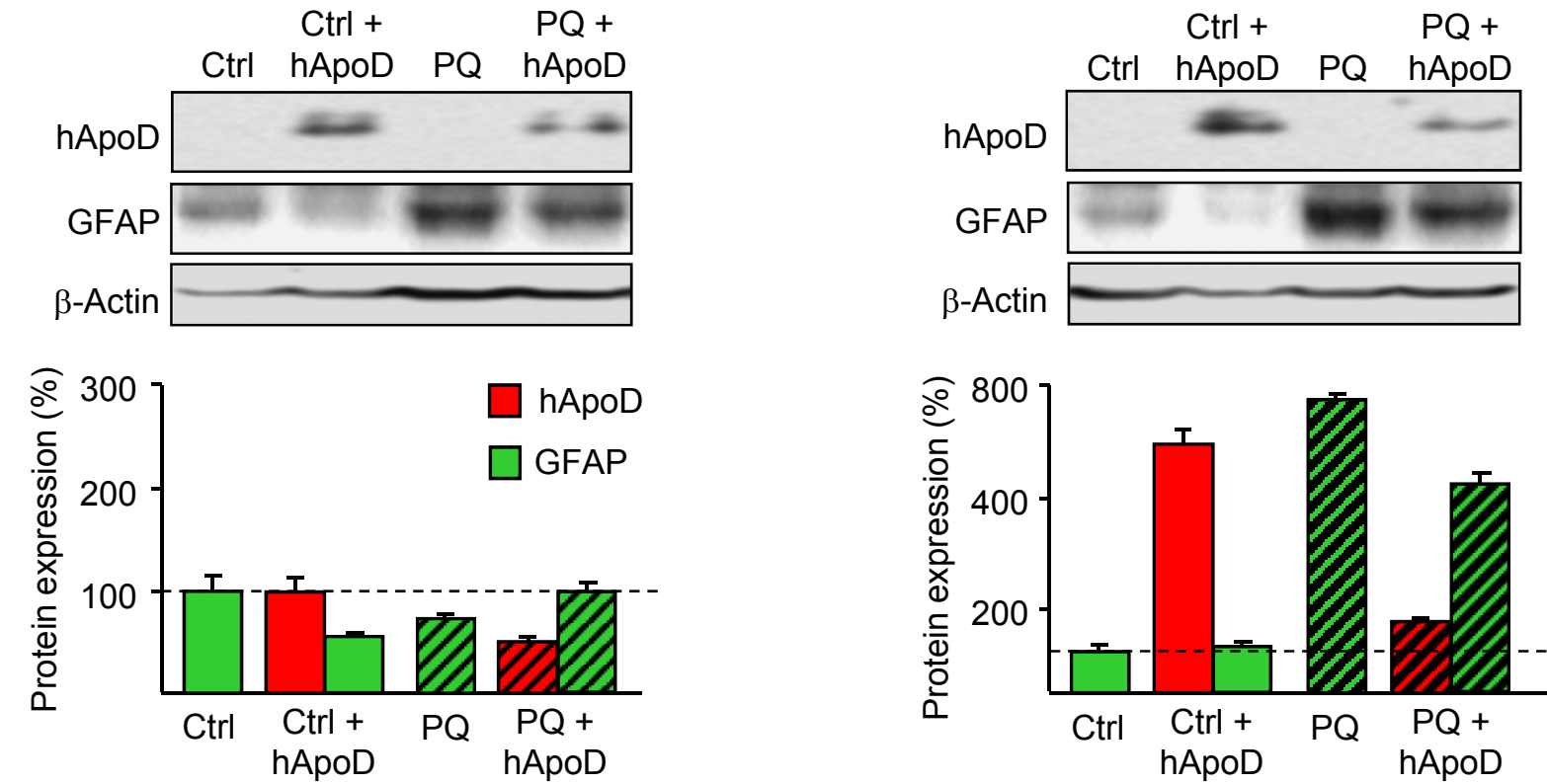
Table 1

WT

ApoD-KO

\begin{tabular}{|l|r|r|}
\hline & \multicolumn{1}{|c|}{ Fold change } & \multicolumn{1}{c|}{ Fold change } \\
\hline II19 & $462.08 \pm 83.6$ & $21.09 \pm 6.3$ \\
\hline Ptgs2 & $70.88 \pm 6.6$ & $18.25 \pm 4.7$ \\
\hline Duox1 & $11.06 \pm 1.3$ & $3.55 \pm 0.3$ \\
\hline Srxn1 & $8.22 \pm 1.1$ & $4.34 \pm 0.1$ \\
\hline Sod2 & $3.62 \pm 0.8$ & $1.11 \pm 0.3$ \\
\hline Gpx3 & $2.34 \pm 0.2$ & $-2.31 \pm 0.6$ \\
\hline Sod3 & $2.32 \pm 0.5$ & $-2.26 \pm 0.6$ \\
\hline Mpp4 & $2.31 \pm 0.4$ & $1.07 \pm 0.1$ \\
\hline Aqr & $-1.05 \pm 0.1$ & $-2.67 \pm 0.9$ \\
\hline Noxo1 & $-1.39 \pm 0.2$ & $-4.21 \pm 1.2$ \\
\hline Nos2 & $-1.76 \pm 0.1$ & $-3.43 \pm 0.9$ \\
\hline Nox4 & $-1.87 \pm 0.2$ & $-3.91 \pm 0.8$ \\
\hline Gab1 & $-1.99 \pm 0.1$ & $-5.56 \pm 2.0$ \\
\hline Idh1 & $-2.83 \pm 0.1$ & $-4.41 \pm 1.2$ \\
\hline Epx & $-2.88 \pm 0.3$ & $1.07 \pm 0.5$ \\
\hline Gpx2 & $-5.08 \pm 1.2$ & $-1.32 \pm 0.1$ \\
\hline Lpo & $-5.78 \pm 0.9$ & $-2.01 \pm 0.5$ \\
\hline Ptgs1 & $-70.85 \pm 13.1$ & $-39.43 \pm 13.7$ \\
\hline
\end{tabular}


Figure S1
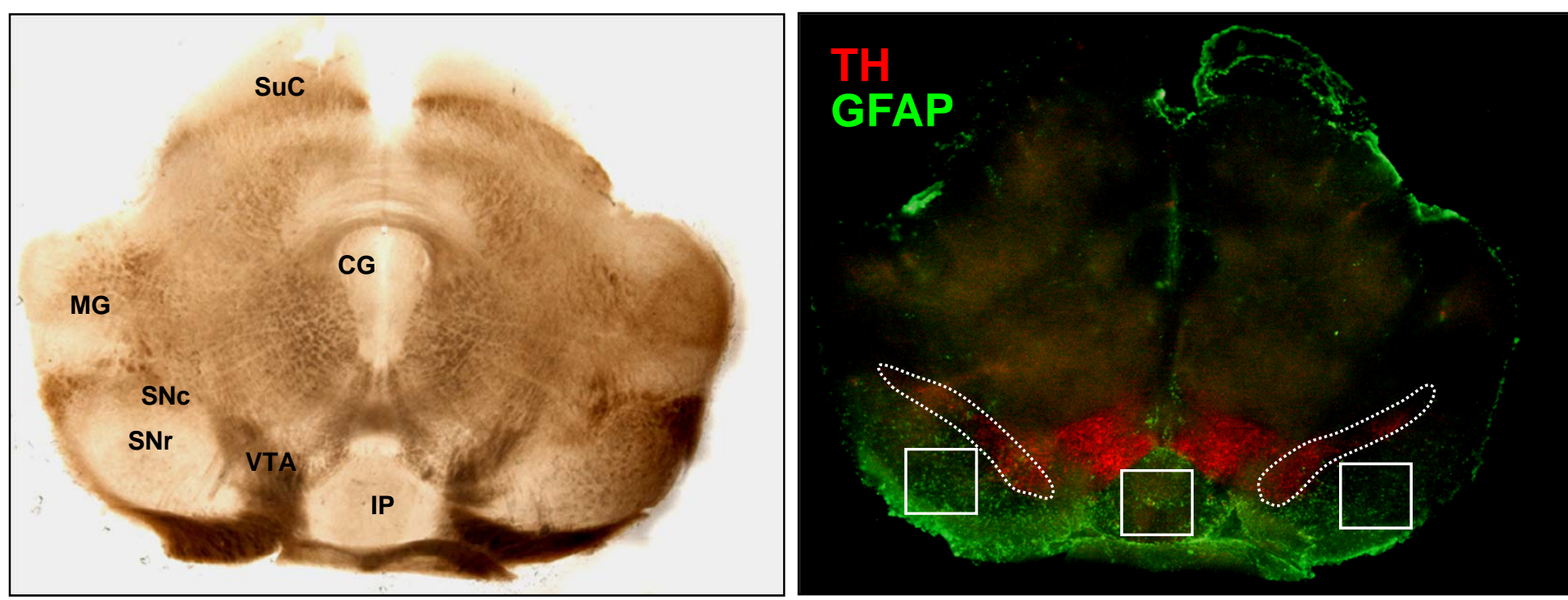
Figure S2

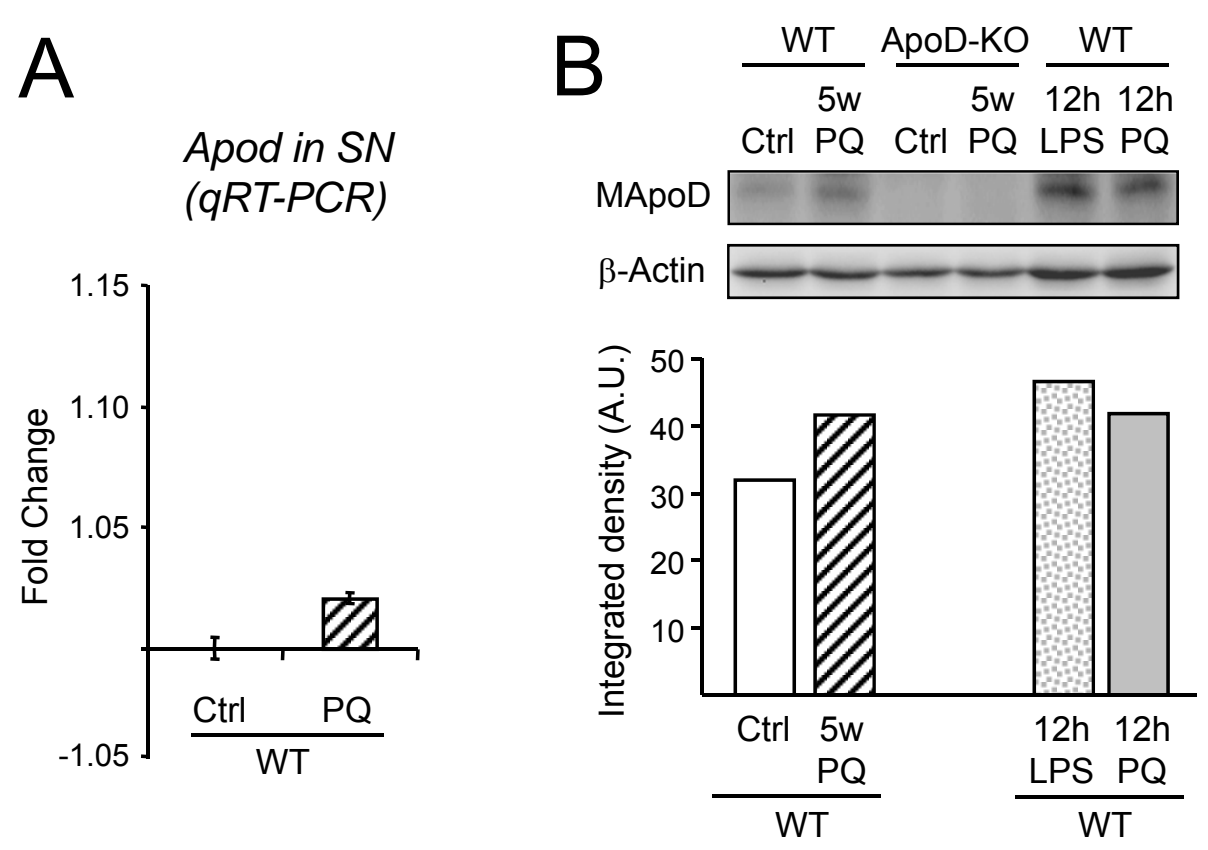


Figure S3

A ApoD time course of expression (qRT-PCR) B
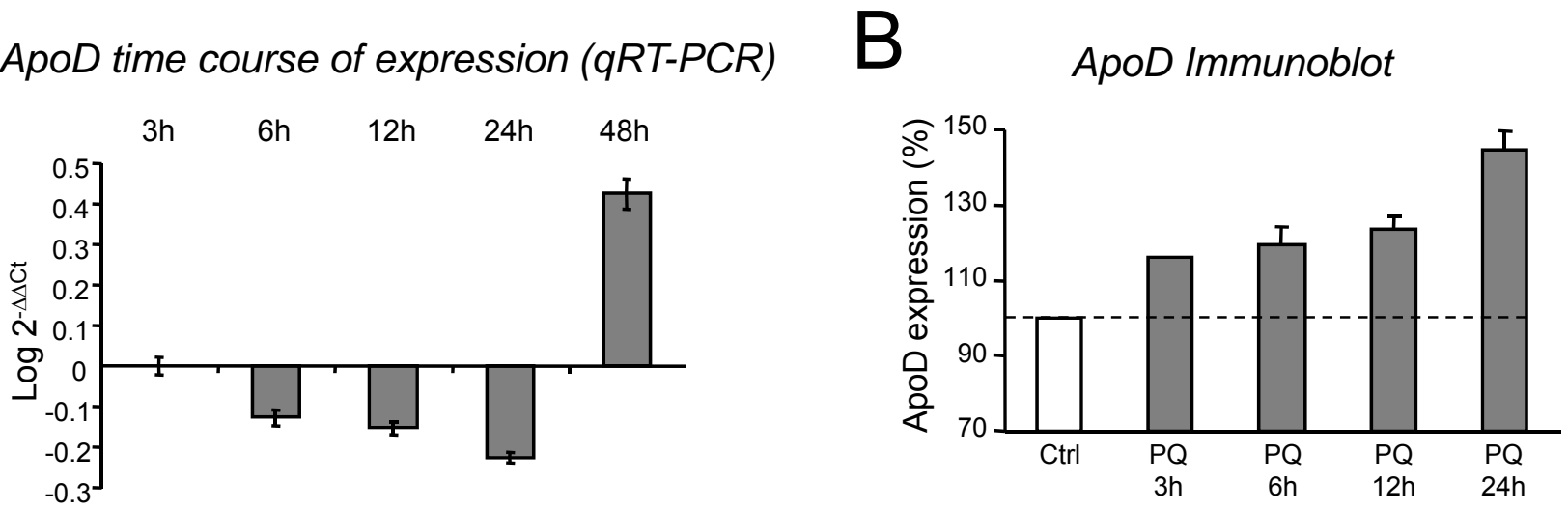
Figure S4

A Apoptosis assay (TUNEL)

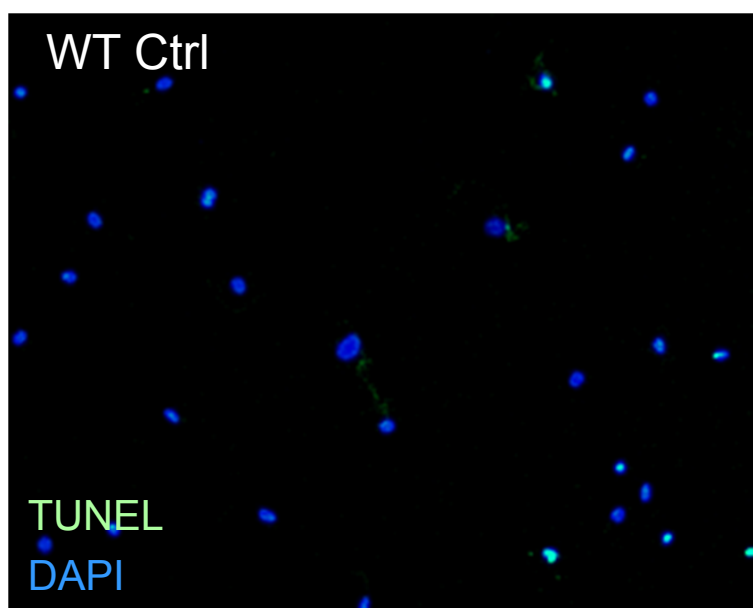

ApoD-KO Ctrl

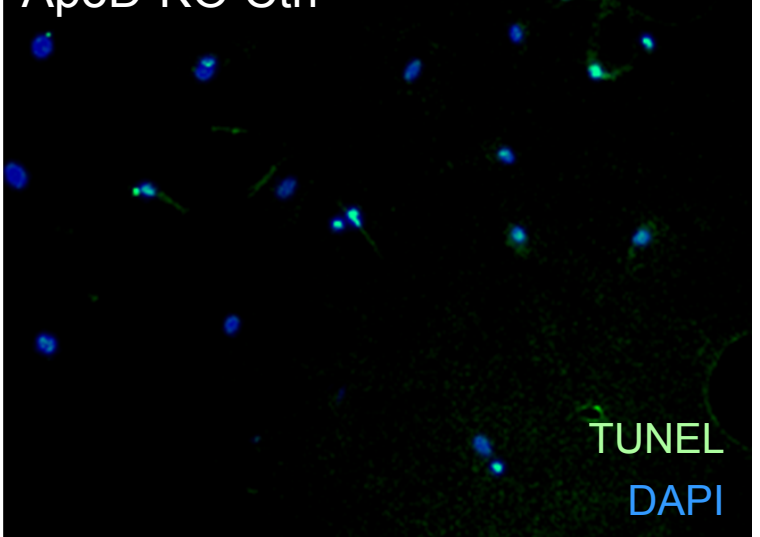

WT $P Q$

TUNEL

DAPI

ApoD-KO PQ

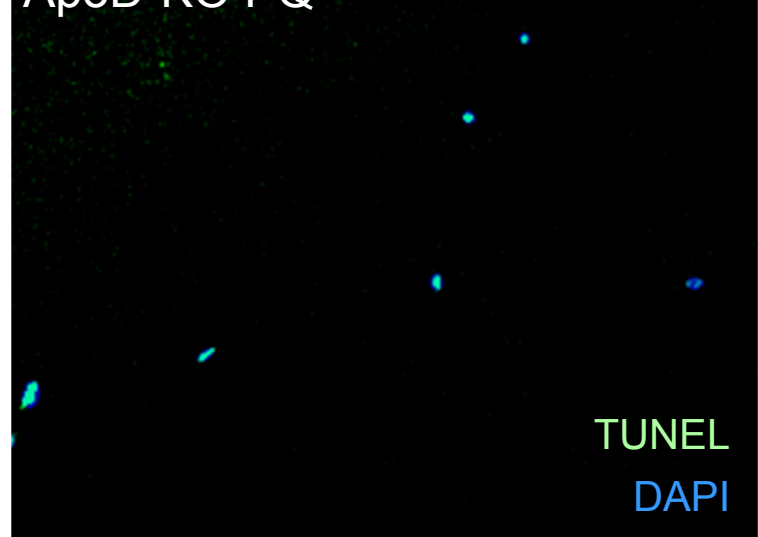

B

Positive control Macrophages + DNasel

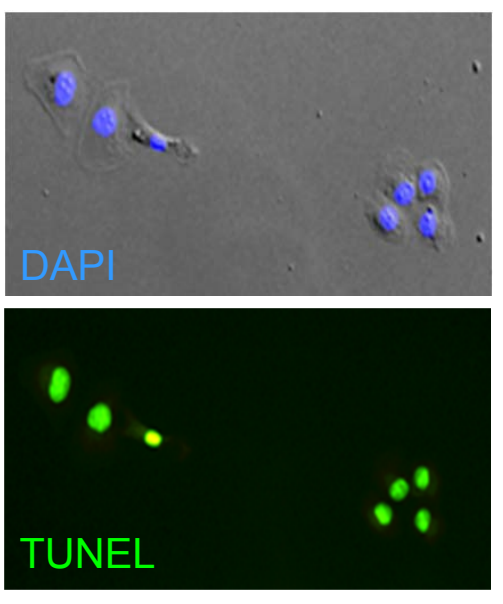

C

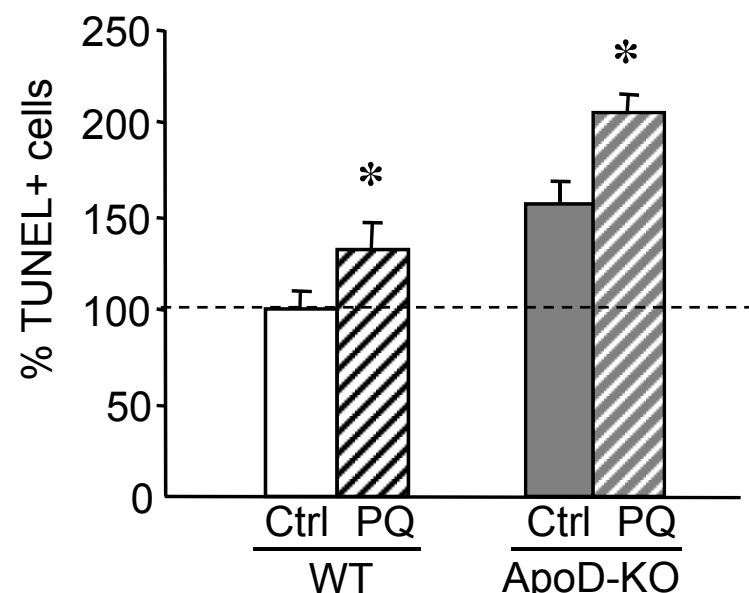


Figure S5

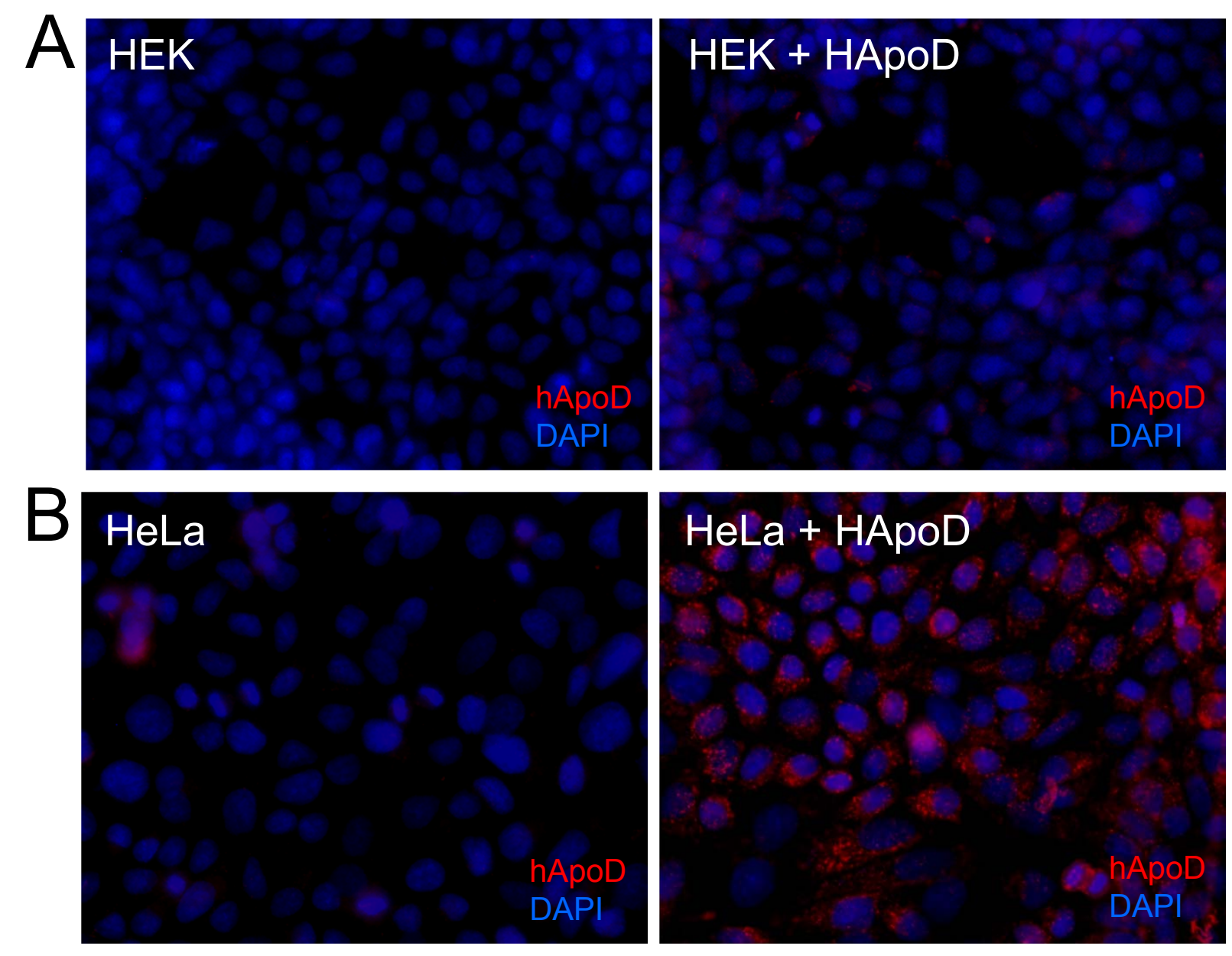


Figure S6

Gene pathways (GNCPro) analysis
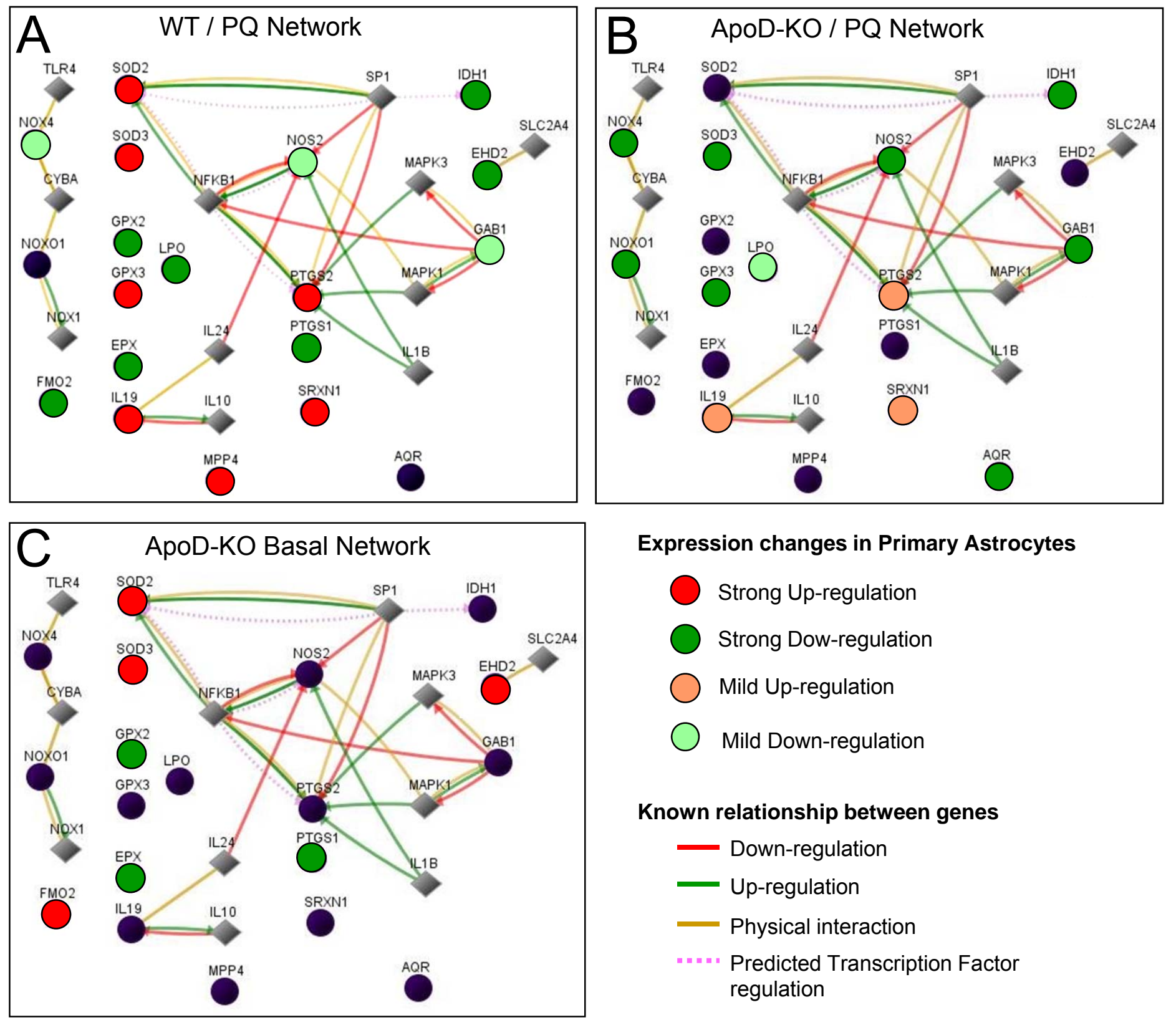

Expression changes in Primary Astrocytes

Strong Up-regulation

Strong Dow-regulation

Mild Up-regulation

Mild Down-regulation

Known relationship between genes

$$
\begin{aligned}
& \text { — Down-regulation } \\
& \text { Up-regulation } \\
& \text { — } \text { Physical interaction } \\
& \text { - . . Predicted Transcription Factor } \\
& \text { regulation }
\end{aligned}
$$


Table S1

\begin{tabular}{|l|r|}
\hline & \multicolumn{1}{|c|}{ WT } \\
\hline & Fold change \\
\hline II19 & $462.08 \pm 83.60$ \\
\hline Ptgs2 & $70.88 \pm 6.65$ \\
\hline Txnrd1 & $22.74 \pm 1.66$ \\
\hline Duox1 & $11.06 \pm 1.30$ \\
\hline Srxn1 & $8.22 \pm 1.13$ \\
\hline Xirp1 & $4.00 \pm 1.03$ \\
\hline Sod2 & $3.62 \pm 0.79$ \\
\hline Tmod1 & $2.92 \pm 0.31$ \\
\hline Zmynd17 & $2.77 \pm 0.46$ \\
\hline Gpx3 & $2.34 \pm 0.17$ \\
\hline Sod3 & $2.32 \pm 0.53$ \\
\hline Mpp4 & $2.31 \pm 0.45$ \\
\hline Ehd2 & $-2.21 \pm 0.11$ \\
\hline Ccs & $-2.44 \pm 1.56$ \\
\hline Prdx4 & $-2.55 \pm 0.26$ \\
\hline Nudt15 & $-2.67 \pm 0.28$ \\
\hline Dnm2 & $-2.83 \pm 0.32$ \\
\hline ldh1 & $-2.83 \pm 0.18$ \\
\hline Epx & $-2.88 \pm 0.46$ \\
\hline Gstk1 & $-3.24 \pm 0.35$ \\
\hline Tpo & $-3.25 \pm 0.97$ \\
\hline Ctsb & $-3.32 \pm 0.28$ \\
\hline Apole & $-3.42 \pm 0.20$ \\
\hline Recql4 & $-3.45 \pm 1.19$ \\
\hline Slc41a3 & $-3.46 \pm 0.24$ \\
\hline Slc38a1 & $-3.54 \pm 0.22$ \\
\hline Kif9 & $-3.96 \pm 0.46$ \\
\hline Gpx2 & $-5.08 \pm 1.61$ \\
\hline Ift172 & $-5.29 \pm 0.69$ \\
\hline Nox1 & $-5.39 \pm 1.77$ \\
\hline Lpo & $-5.78 \pm 1.15$ \\
\hline Cyba & $-6.40 \pm 0.56$ \\
\hline Aass & $-15.29 \pm 4.12$ \\
\hline Ncf2 & $-17.84 \pm 1.62$ \\
\hline Txnip & $-22.79 \pm 2.51$ \\
\hline Fmo2 & $-25.79 \pm 2.40$ \\
\hline Ptgs1 & $-70.85 \pm 16.12$ \\
\hline
\end{tabular}

